\title{
Hacia un censo completo unificado de los ejem- plares conservados de Celestina (II): ejemplares de las ediciones de las traducciones (y tres adaptaciones al inglés) localizables en línea ${ }^{1}$
}

\author{
Amaranta Saguar García \\ Universidad Complutense de Madrid
}

\section{RESUMEN}

Aplicando los mismos principios que en la primera revisión censal de los ejemplares conservados de las ediciones de Celestina en castellano a las ediciones de sus traducciones a lenguas europeas (italiano, alemán, francés, holandés, neolatín e inglés) de los siglos XVI a XVIII, en este trabajo consigno los ejemplares conservados localizables en línea a 1 de noviembre de 2021.

Palabras Clave: Censo de ejemplares; Censo de ediciones; Traducciones

\section{Towards an Unified Census of the Existing Copies of Celestina (II): Online Traceable Copies of Editions of its Translations (and Three Adaptations into English)}

\section{ABSTRACT}

By applying the same principles used in the first census of the surviving copies of the editions of Celestina in Spanish to the editions of its translations into European languages (Italian, German, French, Dutch, Neolatin and English) of the $16^{\text {th }}$ to the $18^{\text {th }}$ centuries, in this piece of work I record their online traceable surviving copies, as of the $1^{\text {st }}$ of November 2021.

KeY WORDs: Census of Copies; Census of Editions; Translations

1.- Este artículo forma parte de las tareas preliminares del proyecto La tradición iconográfica de Celestina: materialidad y recepción de las ediciones ilustradas en la Edad Moderna (IcoCel), dentro del Programa de Atracción de Talento de la Comunidad de Madrid (2018-T1/HUM-11717), y de los materiales de trabajo del proyecto Parnaseo. Servidor Web de Literatura Española, (FFI201782588-P AEI/FEDER, UE). 
En 2020 vio la luz en esta misma revista el primer censo de ejemplares conservados de las ediciones en castellano de Celestina localizables en línea (Saguar García 2020). Apenas un año después se han confirmado las peores —o mejores, según como se mire- previsiones del mismo y está ya obsoleto: el presente número de Celestinesca dedica uno de sus artículos a un ejemplar de la Biblioteca Estatal de Ulm que, además de no figurar entre los recogidos en el censo, da testimonio de una edición desconocida de la Tragicomedia con la fecha y el lugar de impresión contrahechos "Sevilla 1502", identificada por las autoras como [Roma: Marcello Silber: 1512-1515] (Lacarra y Jiménez Ruiz 2021). ¿Por qué, entonces, emprender también el censo de los ejemplares conservados de las ediciones de las traducciones de Celestina localizables en línea, si ya solo el de los de las ediciones en castellano ha probado que cualquier intento de mantener un censo de los mismos es una tarea digna de Sísifo? Pues porque, al contrario que los ejemplares en castellano, los de sus traducciones están mucho menos controlados.

Probablemente por su posición liminal entre la literatura española y las literaturas de sus respectivas lenguas, que hace que ni los especialistas de la una ni los especialistas de las otras las tengan en consideración como objeto de investigación, las traducciones antiguas de Celestina han recibido una atención desigual por parte de la crítica. La traducción italiana de Alphonso Hordognez es la que, con diferencia, ha sido más atendida debido, sobre todo, a su interés para la tradición textual en castellano. Puesto que la editio princeps de la traducción italiana es el testimonio más antiguo conservado del estado redaccional de la Tragicomedia y, además, ocupa un lugar bastante alto en todos los stemmata que han ido proponiendo los especialistas, ha interesado estudiarla más a fondo, casi siempre en relación con la tradición en castellano. Así, además de de la bastante cuestionable pero, al fin y al cabo, útil edición crítica de Kathleen V. Kish (Hordognez 1973), ha sido el objeto de varias tesis doctorales (Kish 1971; Lampugnani 1983; Lysy 1985; Wagner 1987; Simone 1991; Ubaldi 1993) y trabajos de investigación (p. ej. Scoles 1961; Lampugnani 1992; Melzi 2000; Saguar García 2015), así como, más recientemente, ha merecido ser el tema de algunos trabajos de final de grado (Giraldo 2013; Miragliotta 2017) y monografías (Lampugnani 2015), cuya relativa abundancia contrasta con la muy poca bibliografía específica que, en comparación, ha generado el resto de traducciones antiguas de Celestina. De hecho, es la única traducción que cuenta con sus propios repertorios bibliográficos independientes (Scoles 1964; Morreale 1990; Piñero 1984; Miguel y Canuto 2003) y, por lo tanto, con listas de ejemplares supervivientes más o menos exhaustivas, aunque deudoras de las limitaciones de su tiempo.

Las del resto, si es que las hay, son las de los estudios pre- o posliminares de sus respectivas ediciones académicas modernas. Este es el caso de la lista de ediciones y ejemplares localizados de las traducciones francesas 
quinientistas y seiscentistas del primer apéndice de la edición de Gerard J. Brault de la primera traducción anónima francesa de Celestina (Brault 1963: 213-218) y el de la nota bibliográfica sobre las ediciones de la traducción francesa de Jacques de Lavardin de la introducción a la edición de la misma de Denis L. Drysdall (Lavardin 1974: 31-34). También lo es de la sección A del capítulo tres del estudio introductorio de Kathleen V. Kish y Ursula Ritzenhoff a su edición facsimilar de las dos traducciones de Christof Wirsung al alemán (Wirsung 1984: 16-19) y de las descripciones codicológicas de las mismas de la tesis doctoral de Fernando Carmona (2007: 97-107 y 250-252), e, incluso, de la sección mínima dedicada a las ediciones de la traducción anónima holandesa de la introducción a la edición académica de esta última de Lieve Behiels y Kathleen V. Kish (2005: 21-22). Por contra, no hallamos información sobre los ejemplares conservados en la edición de la traducción neolatina de Kaspar von Barth de Enrique Fernández (Barth 2006) ni en las de la traducción inglesa de James Mabbe (1972; 1987; 2013), a veces ni tan siquiera sobre el ejemplar que se ha seguido para transcribir el texto. En cuanto a las ediciones de las adaptaciones escénicas de John Rastell, John Stevens y John Savage, que, sin ser propiamente traducciones de Celestina al inglés, incluyo en el censo por su interés a la hora de estudiar la recepción de la Tragicomedia en las islas británicas, la edición de Antonio López Santos y Rubén Tostado González de la primera solamente da importancia a que se conserva en un ejemplar único (véase la introducción a Rastell 2001: 12), mientras que las segundas no han sido - por lo que yo sé- más que comentadas en la tesis doctoral de Jeremy Newton (1974), donde tampoco hay una lista de ejemplares o una discusión bibliográfica de las diferentes ediciones. Consecuentemente, en pleno año 2021, la información disponible sobre las ediciones y los ejemplares supervivientes de las traducciones quinientistas, seiscentistas y setecentistas de Celestina no es mucho mayor que la del repertorio centenario de Eugenio Krapf (1900), así como también adolece de todas las limitaciones que había en los años sesenta, setenta y ochenta — décadas a las que pertenecen mayoritariamente los estudios citados- para llevar a cabo este tipo de investigaciones bibliográficas².

2.- Obviamente, los que cito no son los únicos estudios que aportan información sobre las ediciones y los ejemplares supervivientes de las traducciones de la Tragicomedia en tiempos de la imprenta manual. El investigador encontrará información relevante al respecto en trabajos no dedicados específicamente a este fin: en estudios dedicados a las ediciones de Celestina, traducidas o no, conservadas en colecciones concretas (p. ej. Penney 1954; Berndt-Kelley 1988; Olivetto 1998; Beardsley 2005; Paolini 2010), en descripciones de ejemplares concretos (p. ej. Givanel Mas 1919; Fernández González 2005) o de la trayectoria editorial de la obra (p. ej. Beardsley 1981; Infantes 2007), en trabajos dedicados a las traducciones de la Tragicomedia en particular (p. ej. Brault 1960; Drysdall 1970; Kish y Ritzenhoff 1980; Kish 1989; Serrano 2008) o en general (p. ej. Menéndez y Pelayo 1943 [1907]; Baldelli 1950; Genske 1978; Gómez Blanco 2001) y, de manera muy resumida, en la «Bibliografía de La Celestina» de Rafael Cornejo (1977) y la «Historia de la recepción de Celestina» de Joseph T. Snow (2001). 
Con la finalidad de proporcionar a los investigadores una información más actualizada y completa, para reunir este censo he usado el mismo método que utilizara en el censo anterior, el de los ejemplares de las ediciones en castellano. A partir de la información extraída de los estudios recién citados, contabilicé todas las ediciones cuya existencia está probada (13 para la traducción italiana de Alphonso Hordognez, 1 por cada una de las traducciones de Christof Wirsung al alemán, 4 para la primera traducción anónima francesa, 6 para la francesa de Jacques de Lavardin, 4 para la traducción anónima holandesa, 1 para la traducción neolatina de Kaspar von Barth, 2 para la traducción al inglés de James Mabbe, aunque en realidad se trata de la misma en los dos casos, y 1 para cada una de las adaptaciones escénicas de John Rastell y John Savage, y 2 para la de John

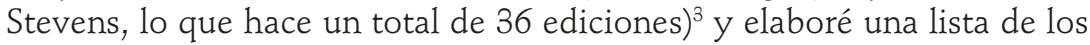
ejemplares localizados, que resultó ser inusualmente breve y más tratándose de una obra como Celestina. Por esta razón, decidí completarla con la información del Universal Short Title Catalogue (USTC) y del repertorio equivalente para el lugar de impresión de cada traducción (o adaptación): el Censimento nazionale delle edizioni italiane del XVI secolo (EDIT16) para la italiana, el Verzeichnis der im deutschen Sprachbereich erschienenen Drucke des 16. Jahrhunderts (VD16) para las dos alemanas, el Verzeichnis der im deutschen Sprachbereich erschienenen Drucke des 17. Jahrhunderts (VD17) para la neolatina, impresa en Frankfurt; la Bibliographie des éditions parisiennes $d u$ 16 e siecle (BP16) para las dos traducciones francesas y, adicionalmente, French Vernacular Books (Pettegree et al. 2007) para las ediciones no parisinas, el Short Title Catalogue Netherlands (STCN) para la traducción holandesa y el English Short Title Catalogue (ESTC) para la inglesa y las tres adaptaciones escénicas de, respectivamente, Rastell, Stevens y Savage.

Con un número de ejemplares de partida mucho más razonable, procedí a comprobar individualmente cada uno de ellos en el catálogo en línea de la biblioteca correspondiente. En el caso de los ejemplares que no figuran en el catálogo en línea que les corresponde o que forman parte de colecciones sin catálogo digital, los busqué en todos los catálogos colectivos nacionales a mi alcance, a través del metacatálogo Karlsruher Virtueller Katalog (KVK) primero y de los propios catálogos individuales después y, cuando no aparecían en estos, también en fuentes escritas o me comuniqué directamente con el personal de las bibliotecas, cuya ayuda ha sido fundamental para elaborar este censo. Asimismo, aproveché cada búsqueda para rastrear cada catálogo, colectivo o particular, digital o impreso, en busca de otros ejemplares aún no consignados en mis fuentes, si bien he de reconocer que, por limitaciones de tiempo, en esta

3.- Como se puede comprobar, en este cómputo no se tienen en cuenta las ediciones de la segunda traducción anónima francesa, la incluida en las ediciones bilingües castellano-francés del siglo XVII, ya que los ejemplares supervivientes de esta ya quedaron consignados en el primer censo bajo los identificadores Pam1633, Rua1633, Rua1633-1634 y Rua1644. 
ocasión no he podido ser tan exhaustiva como en el censo de ejemplares de las ediciones en castellano. Por último, efectué también toda una serie de búsquedas libres, ceñidas al rango cronológico de la imprenta manual, con diferentes cadenas de búsqueda adaptadas a cada una de las traducciones (y adaptaciones) de Celestina: "Tragicocomedia», "Calisto e Melibea», "Célestine», "Calisto et Melibée», "Spanish bawd»...

Estas consultas han localizado un total de 208 ejemplares no recogidos por el USTC ni por ninguno de los repertorios equivalentes listados más arriba: 120 para la traducción italiana de Alphonso Hordognez, 11 para la primera traducción alemana de Christof Wirsung y 4 para la segunda, 3 para la primera traducción anónima francesa, 9 para la de Jacques de Lavardin, 6 para la traducción anónima holandesa, 39 para la traducción neolatina de Kaspar von Barth, 7 para la inglesa de James Mabbe, 5 para la adaptación de John Stevens y 4 para la de John Savage. Esto hace un total de 570 ejemplares — casi 100 más que de las ediciones en castellano, que en 2020 fijé en 476-, descontando 8 catalogados como perdidos o desaparecidos: 253 de la traducción italiana, 21 de la primera traducción de Christof Wirsung y 10 de la segunda, 26 de la primera traducción anónima al francés y 39 de la de Jacques de Lavardin, 15 de la traducción anónima holandesa, 66 de la neolatina de Kaspar von Barth, 80 de la inglesa de James Mabbe, 1 de la adaptación de John Rastell, 24 de la de John Stevens y 36 de la de John Savage. Sin embargo, no han revelado ninguna edición desconocida hasta el momento, excepción hecha de la que evidencia el cuadernillo I del ejemplar con signatura F152.e.2.20 de la Universidad de Cambridge (véase V25b), que ya detecté hace varios años en un proyecto inédito e inconcluso de revisión de los repertorios bibliográficos de la traducción de Alphonso Hordognez (Saguar García 2012) y que, por lo tanto, no supone una novedad. Lo que sí han logrado, en cambio, ha sido arrojar luz sobre las que, a falta de comparación más detallada, parecen ser las emisiones de varias de las ediciones repertoriadas. Así, por ejemplo, resulta que cada una de las traducciones de Christof Wisung al alemán existe en una única edición con dos emisiones, algo que ya habían señalado las editoras - aunque ellas las consideran estados- pero no se refleja en los repertorios pertinentes ni ha tenido mayor repercusión en la crítica. Del mismo modo, la edición de 1631 de la traducción inglesa de James Mabbe existe en dos emisiones, al igual que las dos ediciones de la adaptación de John Stevens, en las que - a falta de un examen más detallado- lo que varía es el librero que figura en el pie de imprenta como vendedor. Esta es también la razón de la multiplicación de emisiones de la edición de la primera traducción anónima francesa de París, Nicolás Barbou, 1492: el pie de imprenta cambia un mínimo de siete veces según la librería a la que se destinara. E incluso VD16 afirma que la traducción neolatina de Kaspar von Barth existe en dos emisiones, pero he de reconocer que, por más que he comparado las imágenes de control, no he sabido ver la diferencia. 
Manteniendo el formato utilizado en el primer censo, cada entrada viene identificada por un número, al que siguen, en este orden, el lugar de impresión, el impresor y la fecha y, en caso de haberlo, el editor, el financiador de la edición, el librero, etc. Después de esta información general se incluye, entre paréntesis, el código abreviado que propongo usar para futuros trabajos sobre las ediciones de las traducciones de Celestina, que tanto refleja los que he utilizado en investigaciones previas sobre las versiones italianas (Saguar García 2012; 2016) y alemanas (Saguar García 2018) como proporciona identificadores nuevos para ediciones que, por lo que me consta, no han tenido uno estable hasta ahora. Tras este paréntesis indico el traductor $y$, a continuación, tras el salto de línea, ofrezco información sobre los principales repertorios digitales específicos que consignan la edición, incluidos los identificadores correspondientes y, de haberlos, los enlaces permanentes a los mismos: además de al USTC, remito a EDIT16 para la traducción italiana, VD16 para las traducciones alemanas, VD17 para la neolatina, BP16 para las traducciones francesas, STCN para las traducciones holandesas y ESTC para la traducción y las adaptaciones escénicas en inglés. Acto seguido, incluyo la información sobre los ejemplares localizados repartida en tres secciones: «Ejemplares localizados con entrada en catálogo en línea», "Ejemplares localizados sin entrada en catálogo en línea» y, por último, "Ejemplares sin asignar, sin localizar o perdidos", que no se tienen en cuenta para ninguno de los cómputos de ejemplares hechos más arriba. Para cada sección añado, entre paréntesis, el número de ejemplares consignados y, para cada ejemplar, proporciono, en este orden, la ciudad, la biblioteca, la signatura enlazada a la entrada pertinente del catálogo en línea (si se dispone de ambos, si no, solamente se proporciona la signatura, sin enlace, o no se proporcionan más detalles) y, entre paréntesis, cualquier comentario sobre el ejemplar que pueda ser de interés, incluidos los enlaces a digitalizaciones. En cursiva señalo los ejemplares que no están recogidos en los repertorios digitales específicos enumerados más arriba.

Finalmente, en este repertorio reincido en mi decisión antibibliográfica de no distinguir por colección ni por biblioteca en los casos de bibliotecas universitarias o bibliotecas con varias sedes, en cuanto que los catálogos enlazados y/o citados proporcionan suficiente información al respecto o es información sencilla de recabar. Añado, además, otra decisión antibibliográfica más, que es la de consignar por separado las emisiones de una misma edición. Esto tiene que ver con que este censo nace con el propósito práctico de permitir a los investigadores localizar de un vistazo los ejemplares que les interesa consultar y, sobre todo, poder organizar sus viajes de investigación más fácilmente. Espero no generar, con esta organización, nuevas ediciones fantasma. 


\section{Ediciones en italiano}

1. Tragicocomedia di Calisto e Melibea, Roma, Eucario Silber, 29 de enero de 1506 (R06): Alphonso Hordognez (trad.)

Principales repertorios on-line: USTC (852859); EDIT16 (CNCE 56102) Ejemplares localizados con entrada en catálogo en línea (9):

- Bolonia, Biblioteca Universitaria, Raro C.91/1 (ejemplar mútilo del primer cuadernillo)

- Cambridge (EE. UU.), Harvard University, *SC5 R6382C Ei506o

- Cracovia, Biblioteca Jagellionska, BJ Cam. K. V. 37

- Londres, British Library, C.62.b.17. (ejemplar digitalizado en Google Books y BL.UK)

- Madrid, Biblioteca Nacional de España, R/39835 (ejemplar mútilo de la hoja final con las coplas posliminares; digitalizado en la Biblioteca Digital Hispánica)

- Milán, Biblioteca Nazionale Braidense, RARIMELZI.065

- Venecia, Biblioteca Nazionale Marciana, DRAMM. 2988. 005

- Trento, Biblioteca Diocesana Vigilianum, dvgg2Y 106 (ejemplar mútilo de la portada)

- San Petersburgo, Российская национальная библиотека [Biblioteca Nacional de Rusia], 6.19.4.87

Ejemplares localizados sin entrada en catálogo en línea (3):

- Cologny, Fondation Martin Bodmer, Spanish Literatur TIV (comunicación personal de Devid Paolini; confirmado con la biblioteca)

- Madrid, Biblioteca de D. Francisco Zabálburu, Vitrina (según el $\mathrm{CCPB}$, ejemplar mútilo de las hojas finales, con hojas manuscritas completando el texto)

- Santander, Biblioteca de Menéndez Pelayo, 30.023 (según el CCPB, ejemplar mútilo de A1, A3-A6 y A8; confirmado en comunicación personal)

2. Tragicocomedia di Calisto e Melibea, Milán, Zanotto [Giovanni] Castiglione, 23 de junio de 1514. Revisada y corregida por Girolamo Claricio (M14): Alphonso Hordognez (trad.)

Principales repertorios on-line: USTC (852862); EDIT16 (CNCE 50034) Ejemplares localizados con entrada en catálogo en línea (12): 
- Cambridge (EE. UU.), Harvard University, **SC5 R6382C Ei5060b

- Génova, Biblioteca Universitaria, RARI H.1.23

- Londres, British Library, 11725.d.13. (ejemplar digitalizado en BL.UK y Google Books)

- Madrid, Biblioteca Nacional de España, R/11303 (ejemplar digitalizado en la Biblioteca Digital Hispánica)

- Milán, Biblioteca Nazionale Braidense, LP. 0044

- New Haven, Yale University, 198081

- París, Bibliothèque Nationale de France, RES P-YG-5

- París, Bibliothèque Nationale de France, 8-BL-16056 BIS

- París, Bibliothèque Nationale de France, 8-RE-6562

- Perugia, Biblioteca Comunale Augusta, ANT I.I 875 (2

- Reggio Emilia, Biblioteca Panizzi, 14.E.23 (ejemplar mútilo de, al menos, la hoja A2)

- Venecia, Biblioteca Nazionale Marciana, DRAMM. 1023

3. Tragicocomedia di Calisto e Melibea, Milán, Oficina Minuciana (Alessandro Minuziano) ${ }^{4}$, enero de 1515. Revisada y corregida por Vincenzo Minuziano, a expensas de Nicolò Gorgonzola (M15): Alphonso Hordognez (trad.)

Principales repertorios on-line: USTC (852871); EDIT16 (CNCE 31058) Ejemplares localizados con entrada en catálogo en línea (12):

- Cambridge (EE. UU.), Harvard University, *SC5 R6382C Ei506oc (ejemplar mútilo de las hojas H5-6, N2, P7)

- Cambridge, University of Cambridge, Norton.e.33

- Florencia, Biblioteca Nazionale Centrale, Th.4.C.402

- Florencia, Biblioteca Nazionale Centrale, Pal.12.5.2.8 (ejemplar mútilo del cuadernillo $\mathrm{R}$ )

- Forli, Biblioteca Comunale Aurelio Saffi, PIANC SALA P 20/284

- Londres, British Library, 11715.aa.9. (ejemplar digitalizado en Google Books y BL.UK)

- Madrid, Biblioteca Nacional de España, R/1473

4.- Vincenzo Minuziano, revisor y corrector de la edición, aparece en varios repertorios —incluidos los míos propios - como el impresor de la misma, sin embargo, la «Officina Libraria Minutiana» del colofón se refiere siempre al negocio de Alessandro Minuziano, padre de Vincenzo, por lo que procedo a corregir los datos de impresión que he estado usando hasta ahora y a atribuir esta edición a Alessandro como, por otra parte, ya hacen EDIT16 y el USTC. 
- Milán, Biblioteca Sormani, VET.F VET.493

- New Haven, Yale University, 198048 (ejemplar mútilo de la última hoja, en blanco)

- Oviedo, Biblioteca Universitaria, CEA-062

- Pisa, Biblioteca Universitaria, H b.12.1

- Torino, Università degli Studi, Coll T 78 (ejemplar digitalizado en Archive.org y OPAL Libri antichi)

Ejemplares localizados sin entrada en catálogo en línea (6):

- Huesca, Biblioteca Pública del Estado (según el CCPB, sin confirmar)

- Milán, Biblioteca Ambrosiana, S.Q.O.II 36 (según la descripción de Scoles [1964: 216], ejemplar mútilo de las hojas finales; sin confirmación)'

- Milán, Biblioteca Trivulziana, Triv K 1634 (según EDIT16, sin confirmación)

- Orvierto, Biblioteca Pubblica Luigi Fumi (según EDIT16, sin confirmación)

- Padua, Biblioteca Civica (según EDIT16, confirmado en comunicación personal)

- Parma, Biblioteca Palatina (EDIT16, sin confirmar, aunque aparece en el Catalogo Alvisi con signatura KK II 49)

4. Tragicocomedia di Calisto e Melibea, Venecia, s. i., 12 de abril de 1515 (V15): Alphonso Hordognez (trad.)

Principales repertorios on-line: USTC (852864); EDIT16 (CNCE 53741) Ejemplares localizados con entrada en catálogo en línea (13):

- Bolonia, Biblioteca Universitaria, A.5.Tab. 1.K.2. 104/1

- Brescia, Biblioteca Civica Queriniana, 3a.I.XII.38

- Buenos Aires, Biblioteca Nacional, 229 FD

- Cambridge (EE. UU.), Harvard University, *SC5 R6382C Ei506od

- Casale Monferrato, Seminario Vescovile - Biblioteca civica Giovanni Canna, BISEM.7642'

- Florencia, Biblioteca Nazionale Centrale, MAGL.5.7.13

- Florencia, Biblioteca Nazionale Centrale, RARI E.6.6.11

5.- El OPAC SBN italiano consigna un ejemplar mútilo del cuadernillo R en el Sistema Bibliotecario di Milano que sospecho pueda ser este, aunque el enlace remita al ejemplar de la Biblioteca Sormani, que, a juzgar por la entrada del catálogo digital, está completo. 
- Florencia, Biblioteca Nazionale Centrale, PALAT 23.4.4.25 (ejemplar mútilo de la hoja 98, en blanco)

- Londres, British Library, 11725.cc.1.

- Milán, Biblioteca Nazionale Braidense, 25.14.O 0026

- París, Bibliothèque Nationale de France, 8-RE-6563 (ejemplar digitalizado en Gallica)

- Pesaro, Biblioteca Oliveriana, A $06-05-23$

- Trier, Stadtbibliothek Weberbach, G 578: 1 an (ejemplar mútilo de la primera hoja y de las cinco últimas)

\section{Tragicocomedia di Calisto e Melibea, Milán, Ioanne Angelo Scin- zenzeler, 16 de marzo de 1519. Revisada y corregida por Gi- rolamo Claricio (M19): Alphonso Hordognez (trad.)}

Principales repertorios on-line: USTC (852863); EDIT16 (CNCE 31361) Ejemplares localizados con entrada en catálogo en línea (4):

- Forli, Biblioteca Comunale Aurelio Saffi, PIANC SALA P 020285

- Madrid, Biblioteca Nacional de España, R/11194

- Perugia, Biblioteca Comunale Augusta, ANT I. O 69

- Washington, The Folger Shakespeare Library, PQ6427 .I8 1519 Cage

Ejemplares localizados sin entrada en catálogo en línea (1):

- Milán, colección personal de Cesare Segre (confirmado en comunicación personal)

\section{Tragicocomedia de Calisto e Melibea, Venecia, Cesare Arrivabene, 10 de diciembre de 1519 (V19a $)^{6}$ : Alphonso Hordognez (trad.)}

Principales repertorios on-line: USTC (852860); EDIT16 (CNCE 29730)

6.- Las dos ediciones de Venecia, Cesare Arrivabene, 10 de diciembre de 1519 se distinguen, sobre todo, porque una de ellas, la que he llamado V19a, usa dos tacos de ancho de caja de escritura diferentes para ilustrar el texto, mientras que la que he llamado V19b usa esos dos y otro más, que sustituye al correspondiente en los autos IV, V, X y XV. Las dos ediciones son tan similares que, que yo haya podido ver, solamente el OPAC SBN italiano se hace eco de que son diferentes, e incluso yo misma pensaba hasta hace poco que se trataba de un problema de emisión/estado. Sin embargo, una comparación de los ejemplares digitalizados de la Biblioteca de Catalunya, la British Library y la Università degli Studi di Torino (V19a) y la Hispanic Society of America (V19b) ha puesto de relieve que hay toda una serie de diferencias en la composición del texto que confirman que se trata de dos ediciones distintas. Por apuntar unas pocas, en algunos lugares V19b separa los nombres de las dramatis personae ubicados al comienzo de cada auto con punto, mientras que V19a lo hace con dos puntos 


\section{Ejemplares localizados con entrada en catálogo en línea (15):}

- Barcelona, Biblioteca de Catalunya, Toda 1-II-5 (ejemplar digitalizado en Google Books y Memoria Digital de Catalunya)

- Cambridge, University of Cambridge, Norton.e.62

- Dublin, Trinity College, S.1.70

- Ferrara, Biblioteca Comunale Ariostea, L 11.4.3

- Londres, British Library, 11726.aa.20. (ejemplar digitalizado en BL Digital Collections y Google Books)

- Milán, Biblioteca Nazionale Braidense, 25. 15.L.0003

- New Haven, Yale University, 198075

- Piacenza, Biblioteca Comunale Passerini Landi, M.XII.69

- Turín, Università degli Studi di Torino, Coll T 77 (ejemplar digitalizado en Archive.org y OPAL Libri antichi)

- Urbino, Università degli Studi di Urbino, 001 D-03 078

- Valencia, Universitat de València, BH Z-09/047

- Venecia, Biblioteca del Museo Correr, INC. I 116 (aún sin respuesta de la biblioteca)

- Venecia, Biblioteca Nazionale Marciana, RARI VEN. 0699

- Venecia, Fondazione Giorgio Cini, FOAN TES 640

- Wellesley, Wellesley College, P187

Ejemplares localizados sin entrada en catálogo en línea (2):

- Como, Biblioteca Comunale (según EDIT16, pero sin confirmación ni de su presencia en los fondos ni de su pertenencia a esta edición)

- Volterra, Biblioteca Guarnacci, XXVI. 1/2 1 (según EDIT16, confirmado en comunicación personal)

(p. ej. en el auto IV). También saltan bastante a la vista la distinta recurrencia de la abreviatura đ para la preposición de, la de dos tipos distintos de z (z y 3 ) o la de las dos rayas oblicuas $\Leftrightarrow$ para marcar que se corta una palabra a final de línea.

Para una primera asignación de los ejemplares a las ediciones correctas, previa a contactar con las bibliotecas pertinentes, me he basado en algunas diferencias del colofón, en concreto, si la palabra diligentia abrevia o no la $n$, si Venetia empieza o no con mayúscula y si se usan las dos rayas oblicuas para separar cinquecento. Cuando he podido, he examinado en persona o a través de un facsímil digital los ejemplares para determinar a qué edición pertenecen y, en los demás casos, he consultado a las bibliotecas sobre el número de tacos diferentes en el ejemplar. Cuando no he recibido respuesta, lo atribuyo por defecto a V19a y lo señalo debidamente. 


\section{Tragicocomedia de Calisto e Melibea, Venecia, Cesare Arrivabene,} 10 de diciembre de 1519 (V19b): Alphonso Hordognez (trad.)

Principales repertorios on-line: USTC (-); EDIT16 (CNCE 78388)

Ejemplares localizados con entrada en catálogo en línea (10):

- Durham, Durham University, Routh 70.F.23

- Haverford, Haverford College Library, PQ6427 .I8 1519

- Iowa, The University of Iowa, PQ6427 .I8 1519

- Los Ángeles, University of California, Z233.I8 R666c 1519

- Madrid, Biblioteca Nacional de España, R/1434

- Mannheim, Universitätsbibliothek, Mf i 172

- Nápoles, Biblioteca Nazionale Vittorio Emanuele III, S.Q. 21. A 3 (aún sin respuesta de la biblioteca, pero EDIT16 lo asigna a V19b)

- Nîmes, Bibliothèque Carré d'Art, $62664^{7}$

- Nueva York, Hispanic Society of America, PQ6427 .I82 1519 (ejemplar digitalizado en TeXTRed)

- París, Bibliothèque Nationale de France, Rés.p.Yg.6

8. Tragicocomedia di Calisto e Melibea, Venecia, Gregorio de Gregori, noviembre de 1525 (V25a) ${ }^{8}$ : Alphonso Hordognez (trad.)

Principales repertorios on-line: USTC (852865); EDIT16 (CNCE 29742) Ejemplares localizados con entrada en catálogo en línea (27):

- Berna, Universität Bern, ZB Bong VI 93

- Brescia, Biblioteca Queriniana, 5a.F.VIII.7m5

- Cambridge (EE. UU.), Harvard University, *SC5 R6382C Ei506og

- Cambridge, University of Cambridge, Bute.31 (mútilo del folio P1 y de la hoja final en blanco)

- Casale Monferrato, Seminario Vescovile, BISEM.8090'.7

- Ciudad del Vaticano, Biblioteca Apostolica Vaticana, Dramm. Allacci.76(int.4)

7.- Aparece por duplicado en el USTC.

8.- Cuando no he obtenido respuesta de la biblioteca, asigno por defecto a esta edición los ejemplares cuyas entradas en sus respectivos catálogos no dan información sobre el impresor y aquellos que, aun dándola, no permiten distinguir si se trata de un ejemplar de V25a o de V25c, las dos con colofón de Venecia, Gregorio de Gregori, noviembre de 1525. Señalo esta circunstancia en el comentario a los ejemplares afectados, donde también incluyo información sobre la composición, mixta o no del ejemplar (véase la nota 12). 
- Estocolmo, Kungliga Biblioteket, 137 P c Celestina (la entrada del catálogo no permite asignar el ejemplar con seguridad a V25a, V25b o V25c ni determinar si se trata o no de un ejemplar mixto; no he recibido aún respuesta de la biblioteca)

- Florencia, Biblioteca Nazionale Centrale, RARI.Landau Finaly 420./b (ejemplar mixto, el colofón es el de esta edición porque el cuadernillo $\mathrm{P}$ lo es, pero el resto es de una de las ediciones de Francesco Caron, con numeración romana en lugar de arábiga) ${ }^{9}$

- Londres, British Library, C.128.e.17.

- Madrid, Biblioteca Nacional de España, R/8746

- Madrid, Biblioteca Nacional de España, CERV.SEDÓ/8637

- Módena, Biblioteca Estense Universitaria, E 070 G 052001 (ejemplar mixto, los cuadernillos C, D, E, N y P llevan foliación en números romanos, lo que quiere decir que este último es de la edición V25c, ya que lleva el colofón de Gregori)

- New Haven, Yale University, 198073

- Nueva York, Hispanic Society of America, PQ6427 .I82 1525a

- Padua, Biblioteca Universitaria, C.89.c.180

- París, Bibliothèque Nationale de France, 8-RE-6564 (ejemplar mútilo de la hoja F1; no he recibido aún respuesta de la biblioteca sobre su pertenencia a V25a o a V25c ni tampoco sobre si se trata de un ejemplar mixto) ${ }^{10}$

- Roma, Biblioteca Nazionale Centrale, 34.1.F.13 (ejemplar mútilo de la última hoja, en blanco; digitalizado en Google Books)

- Roma, Biblioteca Casanatense, COMM 3311 (ejemplar digitalizado en Google Books)

- San Francisco, State University, PQ6427.I8 1525

- Siena, Biblioteca Comunale degli Intronati, VI/2 O 059

- Stuttgart, Württembergische Landesbibliothek, Fr.D.oct.7841 (la fecha del colofón ha sido manipulada para que indique M.D.LX., como la del ejemplar de Cárcel de amor con el que está encuadernado)

- Turín, Università degli Studi, Coll T 029 (ejemplar digitalizado en OPAL Libri Antichi)

- Turín, Università degli Studi, Coll T 379 (ejemplar mixto, el cuadernillo A es de alguna de las ediciones de Caron; digitalizado en OPAL Libri Antichi)

9.- El USTC y EDIT16 consignan este ejemplar tanto para V25a como para V25b, probablemente debido a esta composición mixta.

10.- El USTC asigna este ejemplar a V25b, pero la entrada del catálogo le atribuye los datos de publicación de «In Vinegia : G. de Gregorii, 1525», por lo que debe tratarse de un error. 
- Urbana, University of Illinois, X 862 R63OCIO1525

- Venecia, Biblioteca Nazionale Marciana, DRAMM. 3707

- Venecia, Biblioteca Nazionale Marciana, DRAMM. 0493

- Verona, Biblioteca Civica, 500 Cinq.F.0551

Ejemplares localizados sin entrada en catálogo en línea (6):

- Milán, Biblioteca Ambrosiana (según Krapf [1900: LXV], sin confirmación)

- Milán, Biblioteca Teatrale Livia Simoni, TS T 20 (confirmado en comunicación personal)

- Padua, Biblioteca Civica (EDIT16, confirmada la presencia en comunicación personal pero sin información sobre si pertenece a la edición V25a o V25c o si se trata de un ejemplar mixto $)^{11}$

- Parma, Biblioteca Palatina (EDIT16, sin confirmar, pero aparece en el Catalogo Alvisi con signatura KK 2 51)

- Roma, Biblioteca dell'Accademia Nazionale dei Lincei e Corsiniana (EDIT16, sin confirmar)

- Praga, Praga, Národni Knihovna České Republiky [Biblioteca Nacional de la República Checa], 9 K 547 (ejemplar digitalizado en Google Books)

Ejemplares no localizados o perdidos (2):

- Berlin, Staatsbibliothek, Xk 2752 (ejemplar desaparecido durante la guerra)

- Copenhague, Det Kongelige Bibliotek, 177:1, 230 (ejemplar perdido desde 1958; sin confirmar su pertenencia a V25a o a V25c, pero con más probabilidades de que pertenezca a la primera)

\section{Tragicocomedia di Calisto e Melibea, Venecia, Francesco Caron, noviembre de 1525 (V25b) ${ }^{12}$ : Alphonso Hordognez (trad.)}

Principales repertorios on-line: USTC (852872); EDIT16 (CNCE 25660)

11.- El USTC y EDIT16 consignan este ejemplar tanto para V25a como para V25b, probablemente debido a esta misma falta de información concreta sobre el ejemplar.

12.- Los catálogos italianos distinguen, sin que haya sido capaz de encontrar el origen de esta distinción, entre una variante $\mathrm{A}$ con la foliación en números romanos, a excepción de los cuadernillos $\mathrm{B}, \mathrm{H}$, I y $\mathrm{N}$, numerados con cifras arábigas; una variante $\mathrm{B}$, cuya foliación está toda en números romanos (y que es la que yo denomino $\mathrm{V} 25 \mathrm{~b}$ ), y una variante $\mathrm{C}$ en todo igual a la B excepto en el colofón, que es de Gregorio de Gregori y no de Francesco Caron (que, por razones prácticas de uso de este listado, es la que yo denomino V25c, a pesar de que, a falta de un examen detallado, la considero emisión de V25b; véase la nota 13). A mi parecer, y a falta de un estudio más sistemático, la variante A no es tal sino que se trata de ejemplares mixtos compuestos de cuadernillos de las ediciones V25b/V25c y V25a en distintas proporciones (véase cómo varía la composición de los diferentes ejemplares con foliación de los dos tipos, que detallo siempre que me ha sido posible comprobarla), pues un examen 


\section{Ejemplares localizados con entrada en catálogo en línea (12):}

- Bérgamo, Biblioteca Civica Angelo Mai, CINQ 2946

- Cambridge, University of Cambridge, F152.e.2.20 (ejemplar mixto, el cuadernillo H lleva numeración arábiga y el cuadernillo I pertenece a una edición a todas luces desconocida pero a plana y renglón de las de 1525, con foliación romana y mismo número de líneas por página pero tipografía cursiva $-y$, por lo tanto, también la caja de texto- ligeramente mayor)

- Estrasburgo, Bibliothèque Nationale et Universitaire, R.100.186,2 (ejemplar mixto, los cuadernillos A-B, F-M y O tienen numeración arábiga)

- Filadelfia, University of Pennsylvania, S-14.4.42

- Filadelfia, University of Pennsylvania, 868 R63C.IO

- Módena, Biblioteca Estense Universitaria, ALPHA H 011016 (ejemplar mútilo de la hoja F8 y de la última página, en blanco)

- Nápoles, Biblioteca Universitaria, Z.A. 0036 (ejemplar mixto)

- Nueva York, Hispanic Society of America, PQ6427.I82 15256 (ejemplar mixto, los cuadernillos A-B, F-M y O llevan numeración arábiga y el resto, romana)

- Rávena, Biblioteca Comunale Classense, F.A. 030006 I

- Turín, Universitá degli Studi, Coll T 079 (ejemplar mixto, los cuadernillos $\mathrm{B}, \mathrm{H}, \mathrm{I}$, K y $\mathrm{N}$ tienen numeración arábiga; digitalizado en OPAL Libri Antichi)

- Venecia, Biblioteca Nazionale Marciana, 55.T.178

- Venecia, Biblioteca del Museo Correr, OP.CICOGNA 0023.3

Ejemplares localizados sin entrada en catálogo en línea (1):

- Verona, Seminario Vescovile (según EDIT16, sin confirmar)

preliminar de los cuadernillos evidencia que los que tienen foliación arábiga reflejan los hábitos compositivos de la edición Gregorio de Gregori (p. ej. la mayor frecuencia de los grupos cultos), así como también se perciben diferencias en el diseño de la conjunción et que apuntan igualmente a de Gregori, aunque haría falta una inspección más detenida para confirmar esta intuición. En todo caso, la existencia de ejemplares mixtos no debe sorprender, pues, al tratarse de dos ediciones a plana y renglón en cursiva de tamaño equivalente y diseño muy similar, sus cuadernillos son perfectamente intercambiables, como demuestran los ejemplares con un único cuadernillo que no pertenece a la misma edición que el resto de la Biblioteca Nazionale Centrale de Florencia o de la Biblioteca Nacional y Universitaria de Estrasburgo y, sobre todo, el ejemplar de la Universidad de Cambridge con un cuadernillo de una edición a plana y renglón distinta de estas de 1525, puesto que la tipografía cursiva es ligeramente mayor, a día de hoy desconocida. La existencia de ejemplares mixtos que no respetan la distribución de los cuadernillos con foliación arábiga de lo que los catálogos italianos han denominado variante $\mathrm{A}$, por lo que he podido ver solamente aplicable a uno de los ejemplares mixtos de la Universidad de Turín - y, aun así, solo admitiendo una errata en la identificación de los cuadernillos, ya que K también está foliado con números arábigos-, refuerza esta impresión. 
10. Tragicocomedia di Calisto e Melibea, Venecia, Gregorio de Gregori, noviembre de 1525 (V25c) ${ }^{13}$ : Alphonso Hordognez (trad.)

Principales repertorios on-line: USTC (N/A); EDIT16 (N/A) Ejemplares localizados con entrada en catálogo en línea (4):

- Barcelona, Biblioteca de Catalunya, Res 1519-12º (ejemplar digitalizado en Google Books)

- Cambridge (EE. UU.), Harvard University, Typ 52525.750

- Princeton, Princeton University, 3176.68.324.8

- Zúrich, Zentralbibliothek, 25.1070

Ejemplares localizados sin entrada en catálogo en línea (1):

- Boston, Public Library, D.170B.5 (según Berndt-Kelley [1988: 20], pero sin confirmar)

11. Tragicocomedia di Calisto e Melibea, [Venecia], Marchiò Sessa, 10 de febrero de 1531 (V31a): Alphonso Hordognez (trad.)

Principales repertorios on-line: USTC (852867); EDIT16 (CNCE 29956) Ejemplares localizados con entrada en catálogo en línea (14):

- Barcelona, Biblioteca de Catalunya, Bon.9-I-8

- Cambridge, University of Cambridge, F153.e.2.7

- Londres, The British Library, G.10159.

- Los Angeles, Getty Research Institute, PQ6427.I8 1531 (ejemplar digitalizado en Archive.org y Hathi Trust)

- Madrid, Biblioteca Nacional de España, CERV.SEDÓ/8638

- Nápoles, Biblioteca Nazionale Vittorio Emanuele III, F.DORIA 3. 27

- Nápoles, Biblioteca Universitaria, G 12933

- Nueva York, Hispanic Society of America, PQ6427.I82 1531a

- Oxford, University of Oxford, ARCH.8o.SP.1531

- París, Bibliothèque Nationale de France, 8-RE-6565 (ejemplar digitalizado en Gallica)

- Roma, Biblioteca Nazionale Centrale, 6. 25.B.52. (ejemplar mútilo

13.- A falta de un examen más sistemático, se trata de una emisión de la edición V25b con colofón de Gregorio de Gregori, no de una edición diferente, ya que no he podido encontrar diferencias al comparar un ejemplar de V25b (el de la Universidad de Pensilvania con signatura S-14.4.42) con otro de V25c (el de la Biblioteca de Catalunya con signatura Res 1519-12): las erratas de foliación son las mismas y, haciendo algunas calas al azar en el texto, no he encontrado diferencias de composición. 
de la portada y de la hoja A8)

- Semur-en-Auxois, Bibliothèque Municipale, I XI 2263

- Toronto, University of Toronto, B-10 00381

- Wolfenbüttel, Herzog August Bibliothek, A: 73.1 Eth

Ejemplares no localizados o perdidos (1):

- Milán, Biblioteca Teatrale Livia Simoni, TS T 14 (según comunicación del personal de la biblioteca, el ejemplar se encuentra perdido desde hace décadas)

12. Tragicocomedia di Calisto e Melibea, Venecia, Francesco di Alessandro Bindoni y Maffeo Pasini, junio de 1531 (V31b): Alphonso Hordognez (trad.)

Principales repertorios on-line: USTC (852873); EDIT16 (CNCE 52382) Ejemplares localizados con entrada en catálogo en línea (8):

- Bolonia, Biblioteca Universitaria, A.5.Tab. 1.K.3. 139/1

- Bolonia, Biblioteca Universitaria, A.5. FF.11. 37.3

- Módena, Biblioteca Estense Universitaria, E 083 D 008 (ejemplar mútilo de las hojas P1, P2, P7 y P8)

- Nueva York, Hispanic Society of America, PQ6427 I82 1543

- París, Bibliothèque Nationale de France, 8-RE-6566 (ejemplar digitalizado en Gallica)

- Reggio Emilia, Biblioteca Panizzi, 15 I 517/1 (signatura del catálogo histórico)

- Siena, Biblioteca Comunale degli Intronati, VI/2 O 069 (ejemplar deteriorado y mútilo de las hojas F1-F5)

- San Petersburgo, Российская национальная библиотека [Biblioteca Nacional de Rusia], 6.17.7.47

Ejemplares localizados sin entrada en catálogo en línea (1):

- Roma, Biblioteca Angelica, O0.8.39/4 (según EDIT16, comprobado en persona) 
13. Tragicocomedia de Calisto e Melibea, [Venecia], Pietro de Nicolini da Sabio, julio de 1535 (V35): Alphonso Hordognez (trad.)

Principales repertorios on-line: USTC (852866); EDIT16 (CNCE 32742) Ejemplares localizados con entrada en catálogo en línea (28):

- Ann Arbor, University of Michigan, PQ 6427 .I8 1535

- Cambridge (EE.UU.), Harvard University, ;'SC5 R6382C Ei506ol

- Cambridge, Trinity College (University of Cambridge), Grylls 6.119

- Cambridge, Clare College (University of Cambridge), y F.8.4 (1)

- Ciudad del Vaticano, Biblioteca Apostolica Vaticana, Stamp. Cappon.V.826

- Detmold, Lippische Landesbibliotekl / Theologische Bibliothek, 02-F 378 a

- Florencia, Biblioteca Nazionale Centrale; PALAT.12.B.A.5.1.20

- Florencia, Biblioteca Nazionale Centrale; RARI.Tordi 711 (ejemplar incompleto)

- Ithaca, Cornell University, PQ6427.19 1535

- Lisboa, Biblioteca Nacional de Portugal, RES. 742 P.

- Madrid, Biblioteca Nacional de España, R/31231

- Madrid, Biblioteca Nacional de España, R/41686

- Madrid, Biblioteca Nacional de España, CERV/SEDÓ 8650

- Manchester, University of Manchester, Walter L. Bullock Book Collection 1719

- Milán, Biblioteca Nazionale Braidense, RACC.DRAM.1978 (ejemplar digitalizado en Ufficio Ricerca Fondi Musicali)

- Múnich, Bayerische Staatsbibliothek, Res/P.o.hisp. 1022 d (ejemplar digitalizado en BSB y Google Books)

- Nápoles, Biblioteca Universitaria, RARI 0342

- New Haven, Yale University, 198074

- Nueva York, Hispanic Society of America, PQ6427.I82 1535

- Oxford, Bodleian Library, Vet. F1 f.51

- París, Bibliothèque Nationale de France, RES- YG- 303

- Piacenza, Biblioteca Comunale Passerini Landi (L) F/2.01.032

- Roma, Biblioteca e Raccolta Teatrale del Bucardo, ED.CINO 180

- San Petersburgo, Российская национальная библиотека [Biblioteca Nacional de Rusia], 6.17.7.48

- Urbana, University of Illinois, X 862 R63OCIO

- Venecia, Fondazione Giorgio Cini, FOAN TES 160

- Venecia, Biblioteca Nazionale Marciana, DRAMM. 3654 
- Washington, The Folger Shakespeare Library, 222-243.1q Ejemplares localizados sin entrada en catálogo en línea (2):

- Milán, Biblioteca Ambrosiana, S.N.X.II.21 (según Scoles [1964: 225], sin confirmar)

- Verona, Biblioteca del Seminario Vescovile (según EDIT16, sin confirmar)

Ejemplares no localizados o perdidos (1):

- París, Bibliothèque Mazarine, 8 44885 (perdido desde 1958)

14. Tragicocomedia de Calisto e Melibea, [Venecia], Giovanni Antonio e Pietro de Nicolini da Sabio, marzo de 1541 (V41): Alphonso Hordognez (trad.)

Principales repertorios on-line: USTC (852870), EDIT16 (CNCE 32352) Ejemplares localizados con entrada en catálogo en línea (41):

- Augsburgo, Universitätsbibliothek, 02/III.12.8.71

- Bochum, Universitätsbibliothek, ERN5154

- Bolonia, Biblioteca Universitaria, A.5.Tab. 1.L.3. 148/1

- Bonn, Universitäts- und Landesbibliothek, Fd 352/8

- Boston, Public Library, XD.541.R63C

- Cambridge, University of Cambridge, XXIII.30.58

- Chiavari, Biblioteca della Società Economica, LET 150 V 4) (ejemplar mútilo de la portada)

- Chicago, University of Chicago, PQ6427.I8O6 1541

- Fermo, Biblioteca Civica Romolo Spezioli, 2 YY 24120

- Filadelfia, University of Pennsylvania, SC5 R6382 Ei541c

- Florencia, Biblioteca Nazionale Centrale, PALAT.12.2.0.1./4.a

- Florencia, Biblioteca Nazionale Centrale, NENC.1.5.6.10

- Forli, Biblioteca Comunale Aurelio Saffi, Raccolte Piancastelli, SCAFF 03606039 (ejemplar mútilo de la hoja A8)

- Friburgo, Universitätsbibliothek, E 1053

- Iowa, University of Iowa, PQ6427.I8 1541

- Londres, British Library, 1072.f.2.

- Londres, British Library, 162.e.53.

- Londres, British Library, 243.a.1.

- Madrid, Biblioteca Nacional de España, R/11567 
- Madrid, Biblioteca Nacional de España, CERV.SEDÓ/8641

- Madrid, Biblioteca Nacional de España, U/6808 (ejemplar digitalizado en la Biblioteca Digital Hispánica)

- Módena, Biblioteca Estense Universitaria, E 083 H 025001

- New Haven, Yale University, 198061

- Nueva York, Public Library, Spencer Coll. Ital. 1541 74-263

- Nueva York, Hispanic Society of America, PQ6427 I82 1541

- Oxford, Bodelian Libraries, $8^{\circ} \mathrm{C} 60$ Art.Seld

- París, Bibliothèque Mazarine, $8^{\circ}$ 44535-1 [Res]

- París, Bibliothèque Nationale de France, YD- 3873

- París, Bibliothèque Nationale de France, RES- YG- 304

- París, Bibliothèque Nationale de France, 8- RE- 6567

- París, Bibliothèque Nationale de France, RESERVE 8- BL- 16056

- París, Université de la Sorbonne, RXVIB 6=30

- Pistoia, Biblioteca Comunale Forteguerriana, L.Sala VI.12.10.9 (ejemplar mútilo de las hojas 105-106 y de las dos últimas)

- Princeton, University of Princeton, 3176.68.324.5

- Turín, Biblioteca dell'Accademia delle Scienze, HJ.VIII.26

- Urbana, University of Illinois, IUA10689

- Valladolid, Biblioteca Universitaria, U/Bc BU 06874 (ejemplar digitalizado en UvaDoc)

- Venecia, Biblioteca Nazionale Marciana, RARI VEN. 0569

- Washington, The Folger Shakespeare Library, PQ 6427. I8 1541 Cage

- Weimar, Herzogin Anna Amalia Bibliothek, Scha BS 4 A 01013 (ejemplar incompleto, solo se conservan los cuadernillos A-H y falta la hoja H8; afectado por el incendio de la biblioteca en 2004)

- Windsor, Eton College, DDi.10.37(01)

Ejemplares localizados sin entrada en catálogo en línea (6):

- Florencia, Biblioteca Marucelliana, 1-00.IX-64 (sin confirmar)

- Lucca, Biblioteca Statale di Lucca, De.160 E.VI.e.32 (no aparece en el catálogo moderno pero sí en el histórico)

- Parma, Biblioteca Palatina (según EDIT16, sin confirmar)

- Roma, Biblioteca Angelica, RR.1.34/1 (según EDIT16, confirmado)

- Roma, Biblioteca Musicale Governativa del Conservatorio S. Cecilia (según EDIT16, sin confirmar)

- San Juan de Puerto Rico, La Casa del Libro (confirmado en comunicación personal) 
15. Tragicocomedia de Calisto y Melibea, Venecia, Bernardino de Bendoni, 1543 (V43): Alphonso Hordognez (trad.)

Principales repertorios on-line: USTC (852874); EDIT16 (CNCE 47620) Ejemplares localizados con entrada en catálogo en línea (15):

- Ciudad del Vaticano, Biblioteca Apostolica Vaticana, Dramm. Allacci.66 int.2

- Filadelfia, University of Pennsylvania, SC5 R6382 Ei505o 1543

- Hartford, Trinity College Library, PQ6427 .173 1543

- Leiden, Universiteitsbibliotheek, 2545 G 27

- Londres, British Library, 1072.f.10.

- Londres, British Library, 243.a.5.

- Madrid, Biblioteca Nacional de España, R/31098

- Madrid, Biblioteca Nacional de España, CERV SEDÓ/8649

- Minneapolis, University of Minnesota, Small Tray 249

- New Haven, Yale University, 198082

- Nueva York, Hispanic Society of America, PQ6427 I82 1543 (el cuadernillo G está mal encuadernado y, por lo tanto, la secuencia de las hojas no es la correcta)

- Oxford, University of Oxford, Douce C 408

- Roma, Biblioteca Nazionale Centrale, 7. 10.G.22 (ejemplar digitalizado en Google Books)

- Venecia, Biblioteca Nazionale Marciana, DRAMM. 3724

- Weimar, Herzogin Anna Amalia Bibliothek, Dd 9: 106 (afectado por el incendio de 2004)

Ejemplares localizados sin entrada en catálogo en línea (1):

- Parma, Biblioteca Palatina (según EDIT16, sin confirmar) 


\section{Ediciones en alemán}

1. Ain hipsche Tragedia von zwaien liebhabenden Mentschen, ainem Ritter Calixstus und einer edlen Junckfrawen Melibea genant, deren Anfang muesam was, das Mittel sieß, mit dem aller bittersten jr bayder Sterben beschlossen, Augsburgo, Sigismund Grimm y Max Wirsung, 20 de diciembre de $1520(\mathrm{AHTa})^{14}$ : Christof Wisung (trad.)

Principales repertorios on-line: USTC (609893); VD16 (R 2930)

Ejemplares localizados con entrada en catálogo en línea (16):

- Berlin, Freie Universität, IN 8274D.520

- Berlin, Staatliche Museen, Kupferstichkabinett, Sign. 2582 (aún sin respuesta de la biblioteca sobre la presencia o no de privilegio imperial al final)

- Berlin, Staatsbibliothek, Xk 2781

- Colonia, Universitäts- und Stadtsbibliothek, AD+S682

- Estocolmo, Kungliga biblioteket, 137 P c Celestina (aún sin respuesta de la biblioteca sobre la presencia o no de privilegio imperial al final)

- Friburgo, Universitätsbibliothek, E 1053,b

- Greifswald, Universitätsbibliothek, 541/Inc. $254^{\circ}$

- Hamburgo, Staats- und Universitätsbibliothek, Inc App A/27 (ejemplar digitalizado en Digitalisierte Bestände)

- Madrid, Biblioteca Histórica Marqués de Valdecillas, BH FLL Res.825 (ejemplar digitalizado en Patrimonio Digital Complutense, Dioscórides y Google Books)

- Marburg, Universitätsbibliothek, 095 XVI C 708 mb

- Múnich, Bayerische Staatsbibliothek, Rar. 662 (ejemplar coloreado, digitalizado en BSB y Google Books)

- Oxford, Bodleian Libraries, Douce VV 30

- París, Bibliothèque Nationale de France, RES-YG-63 (BIS) (aún sin respuesta de la biblioteca sobre la presencia o no de privilegio imperial al final)

- Stuttgart, Württembergischer Landesbibliothek, R 16 Cel 1

- Viena, Österreichische Nationalbibliothek, 58.V.42 ALT PRUNK (ejemplar digitalizado en ÖNB)

14.- A efectos prácticos de este censo, y mientras no se haga una comparación más exhaustiva, sigo la edición de Kish y Ritzenhoff (Wirsung 1984: 16-17) cuando distingue dos estados - aunque yo los considero emisiones-, uno con y otro sin el privilegio "Cum gratia et Priui | legio Imperiali», que yo he denominado respectivamente AHTb y AHTa. Cuando no he podido obtener información sobre la presencia o no de dicho privilegio en el ejemplar, lo atribuyo a AHTa y lo consigno en el comentario correspondiente. 
- Wolfenbüttel, Herzog August Bibliothek, M: L1 206

Ejemplares localizados con entrada en catálogo en línea (1):

- Schweinfurt, Bibliothek Otto Schäfer (según VD16, sin confirmary sin información sobre la presencia del privilegio imperial)

2. Ain hipsche Tragedia von zwaien liebhabenden Mentschen, ainem Ritter Calixstus und einer edlen Junckfrawen Melibea genant, deren Anfang muesam was, das Mittel sieß, mit dem aller bittersten jr bayder Sterben beschlossen, Augsburgo, Sigismund Grimm y Max Wirsung, 20 de diciembre de 1520 (AHTb): Christof Wisung (trad.)

Principales repertorios on-line: USTC (-); VD16 (-)

Ejemplares localizados con entrada en catálogo en línea (3):

- Leipzig, Universitätsbibliothek, 4-B.S.T.39

- London, British Library, C.107.d.4.

- Nueva York, Metropolitan Museum of Art, 31.73.2

Ejemplares localizados con entrada en catálogo en línea (1):

- Cologny, Fundación Martin Bodmer, Span. Lit T-IV (confirmado en comunicación personal)

3. Ainn recht liepliches Buechlin vnnd gleich ain traurige Comoedia (so von den Latinischen Tragicocomoedia genant wirt) darau $\beta$ der Leser vast nutzlichen Bericht von Schaden vnd Gefar fleischlicher Lieb Untrew der Diener Aufsetz der gemaynen Weyber List vnd Geytzigkait der Kupler vnd gleych als inn eynem Spiegel mancherlay Sitten vnnd Aygenschafft der Menschen sehen vnd lernen mag, Augsburgo, Heinrich Steiner, 26 de octubre de $1534(\mathrm{RLBa})^{15}$ : Christof Wirsung (trad.)

Principales repertorios on-line: USTC (608502); VD16 (R 2931)

Ejemplares localizados con entrada en catálogo en línea (6):

15.- Siguiendo una vez más la edición de Kish y Ritzenhoff (Wirsung 1984: 17-18) distingo dos estados - que, de nuevo, considero emisiones-, uno con colofón con la fecha de impresión completa, que yo he denominado RLBa, y otro con solamente el año de impresión, que yo he denominado RLBb. No tengo en cuenta la errata de signatura que Kish y Ritzenhoff consideran característica del estado — pero emisión - con fecha completa por haber encontrado ejemplares de esta sin la errata que podrían haber sido, sencillamente, corregidos durante la impresión, sin que sea necesario recurrir a la existencia de ejemplares mixtos, como hacen las editoras para explicar esta circunstancia. 
- Augsburgo, Staats- und Stadtbibliothek, Rar 74 alias 4 LA 232 (ejemplar manipulado) ${ }^{16}$

- Halle, Universitäts- und Landesbibliothek Sachsen, Dd 409 (ejemplar digitalizado en UH Digitale Bibliothek)

- Hamburg, Staats- und Universitätsbibliothek, A/121924

- Mainz, Stadtbibliothek und Gutenberg-Museum, III m:4/436 a

- Nuremberg, Germanisches Nationalmuseum, [Postinc.] L.1812m

- Viena, Österreichische Nationalbibliothek, 259709-B. Fid

Ejemplares localizados sin entrada en catálogo en línea (2):

- Basilea, Kupferstichkabinett und Bibliothek des Kunstmuseums, KK Inv. 1970.276 (presencia confirmada en comunicación personal)

- Zwickau, Ratsschulbibliothek, 24.8.22.(1) (según VD16, confirmado)

Ejemplares no localizados o perdidos (1):

- Berlín, Staatsbibliothek, 4" Xk 2786 (ejemplar perdido durante la Segunda Guerra Mundial; no es posible saber si pertenece a RLBa o a RLBb)

4. Ainn recht liepliches Buechlin vnnd gleich ain traurige Comoedia (so von den Latinischen Tragicocomoedia genant wirt) darau $\beta$ der Leser vast nutzlichen Bericht von Schaden vnd Gefar fleischlicher Lieb Untrew der Diener Aufsetz der gemaynen Weyber List vnd Geytzigkait der Kupler vnd gleych als inn eynem Spiegel mancherlay Sitten vnnd Aygenschafft der Menschen sehen vnd lernen mag, Augsburgo, Heinrich Steiner, 1534 (RLBb): Christof Wirsung (trad.)

Principales repertorios on-line: USTC (-); VD16 (-)

Ejemplares localizados con entrada en catálogo en línea (2):

- Erlangen, Universitätsbibliothek, H00/R.L 166 da

- Wolfenbüttel, Herzog August Bibliothek, A: 18.10 Eth.

16.- La portada no lleva el pie «Liber ad lectorem I Qui variae varios fortunae discere lusus | Expetit, \& vitae ludicra longae suiae: | Ac Veneris vires alieno scire periclo, I Me legat, expertus cuncta docere volo. I M.D. XXXIIII.» debajo del grabado. En comunicación personal, el director en funciones de la Staats- und Stadtbibliothek Augsburg Berthold Kreß me ha confirmado que esta particularidad no tiene que ver con la edición sino que se debe a que la hoja de portada ha sido restaurada con pérdida de esa parte del texto. 


\section{Ediciones en francés}

1. Célestine, en laquelle est traicte des deceptions des serviteurs envers leurs maistres et des macquerelles envers les amoureux, translaté $d^{\prime} y$ talien en françois, París, Nicolas Cousteau, 1 de agosto de 1527. A costa de Galliot du Pré (Par27a) ${ }^{17}$ : Anónimo (trad.)

Principales repertorios on-line: USTC (21038); BP16 (105507)

Ejemplares localizados con entrada en catálogo en línea (6):

- Berlín, Kunstbibliothek, Gris 1370 kl

- Buenos Aires, Biblioteca Nacional Mariano Moreno, 70 BIS FD

- Cambridge (EE. UU.), Houghton Library, *SC5 R6382C Eh527c

- París, Bibliothèque Nationale de France, RES-YG-307 (ejemplar digitalizado en Gallica)

- París, Bibliothèque Nationale de France, Rothschild 3059 (1474 a) $=\mathrm{IV}, 8,36$

- Poitiers, Médiathèque François Mitterrand, DR 229

Ejemplares localizados con entrada en catálogo en línea (1):

- Cologny, Fundación Martin Bodmer (según BD16, sin confirmar)

2. Célestine, en laquelle est traicte des deceptions des serviteurs envers leurs maistres et des macquerelles envers les amoureux, París, Nicolas Cousteau, 1 de agosto de 1527. A costa de Galliot du Pré (Par27b): Anónimo (trad.)

Principales repertorios on-line: USTC (27464); BP16 (105507)

Ejemplares localizados con entrada en catálogo en línea (3):

- Barcelona, Biblioteca de Catalunya, Bon. 9-I-13 (ejemplar digitalizado en Google Books)

- Madrid, Biblioteca Nacional de España, R/1467

- Wolfenbüttel, Herzog August Bibliothek, M: Lm 875a ${ }^{18}$

17.- Denomino Par27a la emisión A del apéndice a la edición de la primera traducción francesa anónima de Brault (1963: 213), con la frase «translaté dytalien en francois» en portada, y Par27b a la emisión B, sin ella.

18.- El USTC duplica los ejemplares de la Herzog August Bibliothek, probablemente por interferencia con el ejemplar de Par78c, que no recoge la entrada correspondiente. 
3. Célestine, en laquelle est traicte des deceptions des serviteurs envers leurs maistres et des macquerelles envers les amoureux, translaté $d^{\prime} y t a l i e n$ en françois, París, Jean Saint-Denis, 19 de noviembre de 1529 (Par29): Anónimo (trad.)

Principales repertorios on-line: USTC (73185); BP16 (106101)

Ejemplares localizados con entrada en catálogo en línea (1):

- París, Bibliothèque Nationale de France, 8-BL-16049 (ejemplar único, digitalizado en Gallica)

4. Célestine, en laquelle est traicte des deceptions des serviteurs envers leurs maistres et des macquerelles envers les amoureux, translaté d'ytalien en françois, Lyon, Claude Nourry, 14 de julio de 1529 (Lyon29): Anónimo (trad.)

Principales repertorios on-line: USTC (73184); BP16 (-)

Ejemplares localizados con entrada en catálogo en línea (3):

- Avignon, Bibliothèque Ceccano, Rés. 205 (ejemplar mútilo de la portada $y$ de las hojas finales) ${ }^{19}$

- Besançon, Bibliothèque Municipale d'Étude et de Conservation, 243106

- París, Bibliothèque Nationale de France, RESERVE 8-RE-6552 (ejemplar digitalizado en Gallica)

5. Célestine, en laquelle est traicte des deceptions des serviteurs envers leurs maistres et des macquerelles envers les amoureux, translaté d'ytalien en françois, Paris, Nicolas Barbou, 1542 ${ }^{20}$. [Para Madeleine Boursette, viuda de François Regnault ${ }^{21}$ (Par42a): Anónimo (trad.)

Principales repertorios on-line: USTC (40163); BP16 (110865)

19. - La entrada del catálogo propone que la edición sea de en torno a 1598, pero, tras haber visto unas pocas imágenes proporcionadas por el personal de la biblioteca, me parece que fuera de la edición Lyon29, aunque haría falta un análisis más detallado para corroborarlo. En cualquier caso, no puede ser de una de las ediciones de finales de siglo, ya que tiene la letra gótica característica de las ediciones de la traducción anónima francesa, que solamente se publican en la primera mitad del siglo Xvi.

20.- Nicolas Barbou imprimió esta edición en, al menos, siete emisiones - a falta de una comparación más detallada-, cada una para un librero, que se diferencian por dónde se indica que están a la venta los ejemplares. En el apéndice a su edición de la primera traducción francesa anónima, Brault (1963: 214-215) solo recopiló cinco, las que yo he llamado Par42b (emisión A), Par42e (emisión B), Par42a (emisión C), Par42f (emisión D) y Par42c (emisión E).

21.- Se infiere que se trata de la emisión destinada a la librería de Madeleine Boursette, 
Ejemplares localizados con entrada en catálogo en línea (3):

- Cambridge (EE.UU.), Houghton Library, *SC5 R6382C Eh527ce

- Filadelfia, University of Pennsylvania, SC5 R6382 Eh542c

- Oxford, Bodleian Libraries, Douce C 394 (según BP16, ya que la entrada del catálogo no incluye información sobre el pie de imprenta)

Ejemplares localizados sin entrada en catálogo en línea (1):

- Moscú, Российская государственная библиотека [Biblioteca Estatal de Rusia] (según BP16, sin confirmar)

6. Célestine, en laquelle est traicte des deceptions des serviteurs envers leurs maistres et des macquerelles envers les amoureux, translaté d'ytalien en françois, París, Nicolas Barbou, 1542. Para Maurice de La Porte (Par42b) 22 : Anónimo (trad.)

Principales repertorios on-line: USTC (40161); BP16 (110865)

Ejemplares localizados con entrada en catálogo en línea (2):

- París, Bibliothèque Nationale de France, RESERVE 8-BL-16050

- Múnich, Bayerische Staatsbibliohek, Res/P.o.hisp. 200 n (ejemplar digitalizado en BSB y en Google Books)

7. Célestine, en laquelle est traicte des deceptions des serviteurs envers leurs maistres et des macquerelles envers les amoureux, translaté $d^{\prime}$ ytalien en françois, Paris, Nicolas Barbou, 1542. Para Pierre Sergent (Par42c) ${ }^{23}$ : Anónimo (trad.)

Principales repertorios on-line: USTC (40159 y 40160); BP16 (110865)

Ejemplares localizados con entrada en catálogo en línea (1):

- Nueva York, Hispanic Society of America, PQ6426 .F72 1542

viuda de François Regnault, en cuanto que el pie de imprenta reza «On les vend a Paris a la grant rue Sainct Jacques deuant leglise des Mathurins a lenseigne de Lelefant». No transcribo los pies de imprenta semipaleográficamente porque no siempre he podido ver la portada y me baso en las entradas de los catálogos que he consultado.

22.- Pie: "On les vend a Paris au clos Bruneau par Morice de La Porte a lenseigne sainct Claude».

23.- Pie: "On les vend a Paris en la rue neufue nostre dame par Pierre Sergent a lenseigne sainct Nicolas». 
8. Célestine, en laquelle est traicte des deceptions des serviteurs envers leurs maistres et des macquerelles envers les amoureux, translaté d'ytalien en françois, Paris, Nicolas Barbou, 1542. Para Jean Il Bonhomme (Par42d) 24: Anónimo (trad.)

Principales repertorios on-line: USTC (-); BP16 (110865)

Ejemplares localizados con entrada en catálogo en línea (1):

- Madrid, Biblioteca de Palacio, I/B/204

9. Célestine, en laquelle est traicte des deceptions des serviteurs envers leurs maistres et des macquerelles envers les amoureux, translaté d'ytalien en françois, Paris, Nicolas Barbou, 1542. Para Jean Foucher (Par42e) ${ }^{25}$ : Anónimo (trad.)

Principales repertorios on-line: USTC (40157) 26 ; BP16 (110865)

Ejemplares localizados con entrada en catálogo en línea (1):

- Londres, British Library, 243.a.6.

10. Célestine, en laquelle est traicte des deceptions des serviteurs envers leurs maistres et des macquerelles envers les amoureux, translaté $d^{\prime}$ ytalien en françois, Paris, Nicolas Barbou, 1542. Para Oudin Petit (Par42f) ${ }^{27}$ : Anónimo (trad.)

Principales repertorios on-line: USTC (40158); BP16 (110865) Ejemplares localizados con entrada en catálogo en línea (2):

- Copenhague, Det Kongelige Bibliotek, 177:1, 23002006

- Madrid, Biblioteca Nacional de España, R/1290528

24.- Pie: «On les vend a Paris a la Rue des Mathurins par Jehan bonhomme a lenseigne de la Caige dor».

25.- Pie: "On les vend a Paris a la grant Rue sainct Jacques par Jehan Foucher».

26.- El USTC consigna dos ejemplares, uno en la Bayerische Staatsbibliothek y otro en la Bibliothèque Nationale de France, para esta edición que no he podido corroborar.

27.- Pie: «On les vend a Paris a la grant Rue saint Jacques par Oudin Petit a lenseigne de la fleur de lis dor».

28.- Es el ejemplar que el USTC consigna para Par42b. 
11. Célestine, en laquelle est traicte des deceptions des serviteurs envers leurs maistres et des macquerelles envers les amoureux, translaté d'ytalien en françois, Paris, Nicolas Barbou, 1542. Para Vivant Gaultherot (Par42g) ${ }^{29}$ : Anónimo (trad.)

Principales repertorios on-line: USTC (66594); BP16 (110865) Ejemplares localizados con entrada en catálogo en línea (1):

- Berna, Universität Bern, MUE Bong VI 169

12. La Celestine, fidellement repurgée, et mise en meilleure forme par Jacques de Lavardin, París, Gilles Robinot, 1578a (Par78a) ${ }^{30}$ : Jacques de Lavardin (trad.)

Principales repertorios on-line: USTC (38424), BP16 (-)

Ejemplares localizados con entrada en catálogo en línea (10):

- Besançon, Bibliothèque municipale, 243107

- Cambridge (GB), University of Cambridge, $\mathrm{O}^{*} 1329$ (G)

- Erlangen, Universität Erlangen-Nürnberg, H00/ R L 166 d

- Mannheim, Universitätsbibliothek, Sch 077/065

- Múnich, Bayerische Staatsbibliothek, P.o.gall. 1201 (ejemplar digitalizado en Google Books y BSB) ${ }^{31}$

- Oxford, University of Oxford, $8^{\circ} \mathrm{C} 139$ Art

- París, Bibliothèque Mazarine, $8^{\circ} 22047$ [Res. N]

- París, Bibliothèque Nationale de France, 8-RE-6553

- París, Bibliothèque Nationale de France, 8-BL-16051

- París, Bibliothèque Sainte-Geneviève, 8 Y 2956 INV 5524 RES

Ejemplares localizados sin entrada en catálogo en línea (1):

- Cologny, Fondation Martin Bodmer (según el USTC, sin confirmar)

29.- Pie: «On les vend a Paris a la grant Rue sainct Jacques Par Viuant Gaultherot a lenseigne sainct Martin».

30.- Esta edición aparece con privilegio real de seis años pero, a la vez, aparecen al menos otras dos ediciones con seguridad muy cercanas en el tiempo en la misma ciudad (P78b y P78c) sin él.

31.- Es el ejemplar que el USTC consigna para Par78c. 
13. La Celestine, fidellement repurgée, et mise en meilleure forme par Jacques de Lavardin, París, Guillaume Chaudière, 1578 (Par78b) ${ }^{32}$ : Jacques de Lavardin (trad.)

Principales repertorios on-line: USTC (38423), BP16 (-)

Ejemplares localizados con entrada en catálogo en línea (1):

- Chantilly, Bibliothèque du Château, XI-F-056

14. La Celestine, fidellement repurgée, et mise en meilleure forme par Jacques de Lavardin, París, Nicolas Bonfons, [¿1578?] $\left(\right.$ Par78c ${ }^{33}$ : Jacques de Lavardin (trad.)

Principales repertorios on-line: USTC (6295 y 61999) 34 , BP16 (-)

Ejemplares localizados con entrada en catálogo en línea (9):

- Cambridge (EE.UU.), Harvard University, *SC5 R6382C Eh577lc

- Londres, British Library, 11726.a.18.

- Madrid, Biblioteca Nacional de España, R/41530

- Moscú, Российская государственная библиотека [Biblioteca Estatal de Rusia], Paris Bonfons [1578] $8^{\circ}$

- París, Bibliothèque Nationale de France, Rés. YG 309

- París, Bibliothèque Nationale de France, 8-BL-16052

- París, Bibliothèque Nationale de France, 8-BL-16053 (ejemplar digitalizado en Gallica)

- Washington, Folger Shakespeare Library, PQ6427 F6 1578 Cage

- Wolfenbüttel, Herzog August Bibliothek, A: 161.1 Eth

32.- Al no haber podido consultar el ejemplar de Chantilly, desconozco si emparienta con Par78a o Par78c, si se trata de una emisión de alguna de las dos o si es una edición completamente diferente.

33.- Después de la traducción de Jacques Lavardin de Celestina, esta edición incluye el poema «La vieille courtisane» de Joachim Du Bellay (1522-1560), que abarca desde el recto de la hoja $\mathrm{Mm} 2$ hasta el verso de la hoja $\mathrm{Nn} 4$. En las hojas que quedan se imprime, además, la «Élegie sur La Célestine de Jacques de Lavardin» de Florent Chrestien (1541-1596), que en la edición con privilegio figura al principio. Esta edición prescinde de los versos latinos «Ad lectorem» de la autorizada. No tiene fecha de impresión, pero la dedicatoria lleva al final el año de 1578, de ahí que se le atribuya convencionalmente esta fecha.

34.- El USTC consigna un ejemplar en la University of Pennsylvania, pero se trata de un microfilm. 
15. La Célestine, tragicomédie traduit d'espagnol en français, ou se voyent les ruses et tromperies dont les maquerelles usent envers les fols amoureux. Dernière édition, Ruan, Theodore, Reinsart, 1598 (Par98): Jacques de Lavardin (trad.)

Principales repertorios on-line: USTC (27525), BP16 (-)

Ejemplares localizados con entrada en catálogo en línea (5):

- Londres, British Library, 243.a.26.

- Múnich, Bayerische Staatsbibliothek, P.o.hisp. 200 p (ejemplar digitalizado en BSB y Google Books) ${ }^{35}$

- Nueva York, Hispanic Society of America, PQ6427 .F72 1598

- París, Bibliothèque Nationale de France, 8-RE-6554

- París, Institut de France, 8 Q Q 740 B * Réserve

Ejemplares localizados sin entrada en catálogo en línea (1):

- Cambridge, Clare College (University of Cambridge), E1 325 (según el USTC, sin confirmar)

16. La Célestine, tragicomédie traduit d'espagnol en français, ou se voyent les ruses et tromperies dont les maquerelles usent envers les fols amoureux. Dernière édition, Rouen, Claude Le Villain, 1598 (Ruan98): Jacques de Lavardin (trad.)

Principales repertorios on-line: USTC (13426), BP16 (-)

Ejemplares localizados con entrada en catálogo en línea (3):

- Copenhague, Det Kongelige Bibliotek, 177:1, $23002007^{36}$

- Gante, Universiteit Gent, BIB.BL.002395/1

- París, Bibliothèque Nationale de France, YG-2662

17. La Célestine, tragicomédie traduit d'espagnol en français, ou se voyent les ruses et tromperies dont les maquerelles usent envers les fols amoureux. Dernière édition, Rouen, Claude Le Villain, 1599 (Ruan99): Jacques de Lavardin (trad.)

35.- El USTC y la edición de la traducción de Jacques Lavardin hablan también de un ejemplar de la Bayerische Staatsbibliothek con signatura P o hisp 38 d que no he sido capaz de localizar en el catálogo digital.

36.- Es el ejemplar que el USTC consigna para Par98. 
Principales repertorios on-line: USTC (27526) ${ }^{37}$, BP16 (-)

Ejemplares localizados con entrada en catálogo en línea (3):

- Londres, British Library, 1072.f.3. (ejemplar digitalizado en BL.UK y Google Books)

- Lyon, Bibliothèque Municipale, 346513 (ejemplar digitalizado en Google Books)

- Nueva York, Hispanic Society of America, PQ6427 .F72 1599

18. La Célestine, tragicomédie traduit d'espagnol en français, ou se voyent les ruses et tromperies dont les maquerelles usent envers les fols amoureux. Dernière édition, Rouen, Claude Le Villain, 1614 (Rua614): Jacques de Lavardin (trad.)

Principales repertorios on-line: USTC (-), BP16 (-)

Ejemplares no localizados o perdidos (1):

- Ejemplar subastado. La única información de la que dispongo es la de esta web: https://www.gazette-drouot.com/lots/10258700.

\section{Ediciones en holandés}

1. Celestina, tragicomedie van Calisto ende Melibea inde welcke staen veel profijtelijcke sententien, ende nootzakelijcke waerschouwinghen, byzonder voor jongheghezellen, betoonende het groot bedroch vande pluym-strijckers, ontrouwe dienaers, ende voor al van de koppelerssen enn lichte vrouwen, T'Hantwerpen, Hans de Laet, 1550 (Hol50): Anónimo (trad.)

Principales repertorios on-line: USTC (407451), STCN (-)

Ejemplares localizados con entrada en catálogo en línea (1):

- Leiden, Rijksuniversiteit Leiden, 1223 H 13 (ejemplar digitalizado en Google Books) 
2. Celestina, tragicomedie van Calisto ende Melibea inde welcke staen veel profijtelijcke sententien, ende nootzakelijcke waerschouwinghen, byzonder voor jongheghezellen, betoonende het groot bedroch vande pluym-strijckers, ontrouwe dienaers, ende voor al van de koppelerssen enn lichte vrouwen, T'Hantwerpen, Heyndrick Heyndricz, [1580, pero 1572-1588] (Hol80) ${ }^{38}$ : Anónimo (trad.)

\section{Principales repertorios on-line: USTC (416006 y 402959), STCN (-)}

Ejemplares localizados con entrada en catálogo en línea (7):

- Amberes, Erfgoedbibliotheek Hendrik Conscience, 754187 [C2-555 i]

- Amberes, Erfgoedbibliotheek Hendrik Conscience, C 14633 [C2531 i]

- Amberes, Museum Plantin-Moretus, A 4229.6

- Gante, Universiteit Gent, BIB.ACC.034938

- La Haya, Koninklijke Bibliotheek, KW 28 D 23 (ejemplar digitalizado en Google Books)

- Londres, British Library, 11726.aa.2.

- Washington, Library of Congress, PQ 6427 .D8 1574 (ejemplar digitalizado en Digital Collections) $)^{39}$

3. Celestina, tragicomedie van Calisto ende Melibea inde welcke staen veel profijtelijcke sententien, ende nootzakelijcke waerschouwinghen, byzonder voor jongheghezellen, betoonende het groot bedroch vande pluym-strijckers, ontrouwe dienaers, ende voor al van de koppelerssen enn lichte vrouwen, T'Hantwerpen, Heyndrick Heyndricz, 1574 (Hol74): Anónimo (trad.)

Principales repertorios on-line: USTC (-), STCN (-)

Ejemplares localizados con entrada en catálogo en línea (2):

- Amberes, Museum Plantin-Moretus, A 3645

- Londres, British Library, C.40.m.9.(10.) (solo se conservan la portada y el privilegio)

38.- Se trata de una edición sin indicación de año, por lo que los catálogos que la recogen suelen dar el rango cronológico 1572-1588 para situarla, que se corresponde con el período de actividad del impresor. Sin embargo, en la crítica celestinesca se ha usado con frecuencia la fecha de 1580, propuesta por Krapf (1900), que mantengo por simple convención. En todo caso, la edición de Behiels y Kish (2005: 22) afirma que la edición se ubica necesariamente entre Hol74 y Hol1616.

39.- El catálogo le atribuye el año de 1574, pero la portada no es la del ejemplar de 1574 de la British Library. A falta de un cotejo sistemático, una comparación de algunas pocas páginas con el ejemplar digitalizado de la Koninklijke Bibliotheek identifica el ejemplar de la Library of Congress como de esta edición. 
Ejemplares no localizados o perdidos (1):

- Berlín, Staatsbibliothek, Xk 2832 (perdido durante la Segunda Guerra Mundial)

4. Celestina, tragicomedie van Calisto ende Melibea inde welcke staen veel profijtelijcke sententien, ende nootzakelijcke waerschouwinghen, byzonder voor jongheghezellen, betoonende het groot bedroch vande pluym-strijckers, ontrouwe dienaers, ende voor al van de koppelerssen enn lichte vrouwen, T'Hantwerpen, Heindryck Heyndricz, 1616 (Hol1616): Anónimo (trad.)

Principales repertorios on-line: USTC (1024496), STCN (317301365)

Ejemplares localizados con entrada en catálogo en línea (5):

- Amberes, Erfgoedbibliotheek Hendrik Conscience, C 1821 [C2-528 i]

- Amsterdam, Universiteit van Amsterdam, OTM: OK 63-2440

- Londres, British Library, 11725.a.7. (ejemplar digitalizado en BL Digital Library y Google Books)

- Madrid, Biblioteca Nacional de España, R/41626

- París, Bibliothèque Nationale de France, 8-YTH-67546

\section{Ediciones en latín}

1. Pornoboscodidascalus latinus, de lenonum, lenarum, conciliatricum, servitiorum, dolis, veneficiis, machinis, plusquam diabolicis, de miseriis juvenum incautorum qui florem, aetatis amoribus inconcessis addicunt; de miserabili singulorum periculo et omnium interitu, liber plane divinus, lingua hispanica ab incerto auctore instar ludi conscriptus Celestinae titulo tot vitae instruendae sententijs, tot exemplis, figuris, monitis, plenus, ut par aliquid nulla fere lingua habeat, Frankfurt, Erben Andreas Wechel, 1624. En casa de David y Daniel Aubry y Clemens Schleich, 1624 (PBD) ${ }^{41}$ : Kaspar von Barth (trad.)

\section{0.- El USTC duplica este ejemplar.}

41.- A pesar de haber comparado las páginas a las que remite VD17 a la hora de afirmar que esta edición existe en dos versiones, no he sido capaz de encontrar la diferencia y, por lo tanto, no he podido controlar los ejemplares en busca de la misma. En consecuencia, trato todos los ejemplares como de la misma edición, a la espera de ser capaz de ver lo que los distingue. 
Principales repertorios on-line: USTC (2135281 y 2138422) $)^{42}$; VD16 $(7: 700585 \text { Q y } 23: 271391 \mathrm{~W})^{43}$

Ejemplares localizados con entrada en catálogo en línea (65):

- Aberdeen, University of Aberdeen, SB 863233

- Albi, Médiathèque Pierre-Amalric, RES. ROCH. 02630

- Aarau, Aargauer Kantonsbibliothek, AKB V 434

- Augsburgo, Staats- und Stadtbibliothek, LA 787

- Berlin, Zentral- und Landesbibliothek, Har 12196

- Braunschweig, Stadtbibliothek, Ministerial M 1275 (8)

- Bristol, University of Bristol, Restricted Small PQ6427

- Bruselas, Koninklijke Bibliotheek België, VH 12.160 A 1 (LP)

- Cambridge, University of Cambridge, Rel.d.62.4

- Cambridge, Trinity College (University of Cambridge), Grylls 1.48[2]

- Cambridge, Trinity College (University of Cambridge), III.6.42[1]

- Cambridge, Trinity College (University of Cambridge), S.26.34[2] (ejemplar mútilo de las páginas 394-462)

- Ciudad del Vaticano, Biblioteca Apostolica Vaticana, Stamp. Barb.O.XI.90

- Copenhague, Det Kongelige Bibliotek, 177:1, 230

- Dillingen, Studienbibliothek Dillingen, Mag/I 2238,1

- Dublín, Marsh's Library, sin signatura

- Durham, University of Durham, Routh 69.I.4/1

- Erfurt, Universitäts- und Forschungsbibliothek Erfurt/Gotha, 03 Lr. $8^{\circ} 00382(02)$

- Erlangen, Universität Erlangen-Nürnberg, Hoo/PHL-VIIII 221 b

- Estocolmo, Kungliga Biblioteket, Signum: 137 P c Polo, G.G. [Diana enamorada] Ero

- Estrasburgo, Bibliothèque Nationale et Universitaire, CD.122.736

- Görlitz, Oberlausitzische Bibliothek der Wissenschaften, A VII $8^{\circ} 16$ y SW III 205

- Gotha, Universitätsbibliothek, 03 - Lr. $8^{\circ} 00382$ (02)

- Göttingen, Niedersächsische Staats- und Universitätsbibliothek, 8 P LAT REC II, 3328 (2) RARA

42.- Los ejemplares que consigna el USTC de la Universidad de Indiana y de la Universidad de Illinois son microfilmes.

43.- El ejemplar de la Staatsbibliothek zu Berlin con signatura an: Xl 1280 que consigna VD17 es un error por otra traducción de Kaspar von Barth, el Pornodidascalus, seu Colloquium muliebre Petri Aretini (1624), que tiene el código de materia Xl 1280. 
- Greifswald, Universität Greifswald, 520/Bh 182

- Halle (Saale), Universitäts- und Landesbibliothek Sachsen-Anhalt, Ha 7643

- Hamburg, Staats- und Universitätsbibliothek, A/300974

- Hannover, Gottfried Wilhelm Leibniz Bibliothek, Bu 139

- Hannover, Gottfried Wilhelm Leibniz Bibliothek, N-A 310

- Jena, Thüringer Universitäts- und LandesbibliothekJena, 8 Bud.Var.179(1)

- Jena, Thüringer Universitäts- und Landesbibliothek Jena, 8 Art.lib.X,2

- Jena, Thüringer Universitäts- und Landesbibliothek Jena, 8 Ph.VIII,20(2)

- Kiel, Universität Kiel, Arch4 216

- La Haya, Koninklijke Bibliotheek, KW 848 C 14

- Londres, British Library, 847.f.17.

- Londres, British Library, 847.k.2.

- Londres, University College London, REF COLLECTIONB STRONG ROOM OGDEN A 130

- Londres, Victoria and Albert Museum, Dyce 8vo 1984

- Londres, Wellcome Library, Closed stores EPB / B EPB/B/1391 (ejemplar digitalizado en EEB)

- Lyon, Bibliothèque Municipale, 346518

- Madrid, Biblioteca Histórica Marqués de Valdecilla, BH MED 3239 (ejemplar digitalizado en Google Books, Dioscórides y la versión antigua de Dioscórides)

- Madrid, Biblioteca Nacional de España, R/11930(2)

- Madrid, Biblioteca Nacional de España, CERV.SEDÓ/5739(1)

- Milán, Biblioteca Nazionale Braidense, TT. 0139 /02

- Moscú, Российская государственная библиотека (Biblioteca Estatal de Rusia), sin signatura

- Múnich, Bayerische Staatsbibliothek, Res/L.eleg.m. 96 (ejemplar digitalizado en BSB y Google Books)

- Nápoles, Biblioteca nazionale Vittorio Emanuele III, V.F. 117 B 63

- Oldenburg, Landesbibliothek, SPR XII 1 4,1

- Oxford, University of Oxford, 8 G 104(2) Art.

- Oxford, University of Oxford, Douce CC 24

- Padua, Biblioteca del Seminario Vescovile, 600.ROSSA.CC.5x.-40

- París, Bibliothèque Mazarine, 8027926

- París, Bibliothèque Nationale de France, YC-9932

- París, Bibliothèque Nationale de France, RES-YG-306 
- Regensburg, Staatliche Bibliothek, 999/Lat.rec.345 (ejemplar digitalizado en BSB y Google Books)

- Rostock, Universität Rostock, Cl-905

- Salzburgo, Universität Salzburg, I 73856

- Stuttgart, Württembergische Landesbibliothek, Fr.D.oct.7842

- Tréveris, Universität Trier, mt48549

- Viena, Österreichische Nationalbibliothek, 74.Y.101 ALT PRUNK (ejemplar digitalizado en ÖNB)

- Wolfenbüttel, Herzog August Bibliothek, 364 Quod. (1)

- Wolfenbüttel, Herzog August Bibliothek, QuN 472 (2)

- Washington, Folger Shakespear Library, 222- 2439 item 2

- Weimar, Herzogin Anna Amalia Bibliothek, 276252 - A

- Wittenberg Lutherstad, Reformationsgeschichtliche Forschungsbibliothek, 8SW1270/1

Ejemplares localizados sin entrada en catálogo en línea (1):

- Blicking, Blicking Hall (National Trust Libraries), 3919

Ejemplares no localizados o perdidos (2):

- Berlín, Staatsbibliothek, Bibl. Diez oct. 7708 (ejemplar perdido durante la guerra)

- Weimar, Herzogin Anna Amalia Bibliothek, 15, 7 : 34 [b] (ejemplar perdido en un incendio)

\section{Ediciones en inglés}

1. A new commodye in Englysh in maner of an enterlude ryght elygant and full of craft of rethoryk, wherein is shewd and dyscrybyd as well the bewte and good propertes of women, as theyr vycys and evy11 condicions, with a morall conclusion and exhortacyon to vertew, [Lond.], J. Rastell, [c. 1530] (INT): atrib. John Rastell (trad.) ${ }^{44}$

Principales repertorios on-line: USTC (515992), ESTC (S119335)

Ejemplares localizados con entrada en catálogo en línea $(1)^{45}$ :

- Oxford, University of Oxford, Mal. 22 (ejemplar digitalizado en EEBO)

44.- No se trata propiamente de una traducción sino de una adaptación escénica abreviada. 45.- El Victoria and Albert Museum conserva, asimismo, una transcripción manuscrita del texto de esta edición bajo la signatura Dyce 25.F.57. 
2. The Spanish bawd, represented in Celestina, or, The tragicke-come$d y$ of Calisto and Melibea: wherein is contained, besides the pleasantnesse and sweetenesse of the stile many philosophicall sentences, and profitable instructions necessary for the younger sort: shewing the deceits and subtilties housed in the bosomes of false servants, and cunny-catching bawds, Londres, John Beale, 1631. Vendido por Robert Allot (Mab1631a) ${ }^{46}$ : James Mabbe (trad.)

Principales repertorios on-line: USTC (3015467 y 3015699) ${ }^{47}$, ESTC (S107195)

Ejemplares localizados con entrada en catálogo en línea (43):

- Austin, University of Texas at Austin, PQ 6427 E56 1631

- Austin, University of Texas at Austin, PQ 6427 E56 1630

- Berkeley, University of California-Berkeley, PQ6427 .E36 1631

- Cambridge, Emmanuel College (University of Cambridge), 301.6 .9 (ejemplar mútilo de A1, en blanco)

- Cambridge, Trinity College (University of Cambridge), VI.12.15 (ejemplar mútilo de A1, en blanco)

- Cambridge (EE.UU.), Harvard University, STC 4911

- Cambridge (EE.UU.), Harvard University, STC 290 (ejemplar mútilo de las páginas 197-202)

- Cambridge (EE.UU.), Harvard University, Keats *EC8.K2262.R622ac

- Cambridge (EE.UU.), Harvard University, Keats *EC8.K2262.Zz634a

- Chicago, University of Chicago, PQ6427.E5M19

- Chicago, Newberry Library, Case folio Y 1565.R64

- Chicago, Newberry Library, Case folio Y 1565 .A362

- Columbus, Ohio State University, PQ6427 .E5 $163^{48}$

- Dresden, Sächsische Staats-, Landes- und Universitätsbibliothek, 1.B.3338, angeb.2 (la entrada del catálogo no da información sobre el pie de imprenta, por lo que no puede saberse si pertenece a Mab1631a o a Mab1631b; aún sin respuesta de la biblioteca)

- Durham (EE.UU.), Duke University, PQ6427 .E56 1631 c.1 (ejemplar mútilo de A1 y $C 6$, en blanco)

46.- Mab1631a y Mab1631b son dos emisiones de la misma edición, una con pie de imprenta «LONDON I Printed by J. B. And are to be sold by I Robert Allot at the Signe of the Beare I in Pauls church-yard. 1631." (Mab1631a) y otra con pie de imprenta "LONDON I Printed by J. B. And are to be sold by I Ralph Mabbe. 1631» (Mab1631b).

47.- El ejemplar de University College que consigna el USTC es en realidad un libro digital. 48.- La entrada del catálogo no deja claro si se trata de un microfilm o de un ejemplar físico. 
- Edimburgo, University of Edinburgh, Df.2.49

- Glasgow, Mitchell Library, 214640 (portada dañada)

- Kansas, University of Kansas, D110

- Leeds, University of Leeds, Brotherton Collection Lt quarto ROJ

- Londres, British Library, 162.m.32.

- Londres, British Library, C.102.K.8 (ejemplar digitalizado en EEBO)

- Londres, King's College London, PQ6427.E5 M1

- Londres, Lamberth Palace, K58.2B/P41E

- Londres, University of London, Bf [Aleman] fol SR

- Lovaina, Katholische Universität, RB1035

- Madrid, Biblioteca Nacional de España, CERV.SEDÓ/8775

- Montreal, Bibliothèque et Archives nationales du Québec, 862.2 R741cem $1631 \mathrm{BMRB}$

- New Haven, Yale University, He43 +22Hm (ejemplar mútilo de A1 y Cc6, en blanco, y con algunas imperfecciones)

- New Haven, Yale University, Osborn fpb40 (ejemplar mútilo de A1 y A2, la primera en blanco y la segunda la portada)

- New Haven, Yale University, Eliz +26

- Oxford, Bodleian Library, Caps. 10.3 (1)

- Oxford, Bodleian Library, C 7.13 Art.

- Oxford, Bodleian Library, Mal. 26 (1)

- Oxford, Bodleian Library, Douce P 741

- Princeton, Princeton University, 17th-740 RHT (según el ESTC pertenecería a Mab1631b, pero la biblioteca ha confirmado que se trata de un ejemplar Mab1631a)

- San Marino (EE.UU.), Huntington Library, 99589

- Sidney, University of Sidney, Macdonald STC 4911

- State College, Penn State University, PQ6427.E56 1631

- Toronto, University of Toronto, Stc 01207

- Urbana, University of Illinois at Urbana-Champaign, Q. 862 R63Oc:Em1631

- Washington, Library of Congress, PQ6427 .E56

- Washington, Folger Shakespeare Library, STC 4911

- York, York Minster, I.F.21(2) 
3. The Spanish bawd, represented in Celestina, or, The tragicke-come$d y$ of Calisto and Melibea: wherein is contained, besides the pleasantnesse and sweetenesse of the stile many philosophicall sentences, and profitable instructions necessary for the younger sort: shewing the deceits and subtilties housed in the bosomes of false servants, and cunny-catching bawds, Londres, John Beale, 1631. Vendido por Ralph Mabbe (Mab1631b): James Mabbe (trad.)

\section{Principales repertorios on-line: USTC (-), ESTC (S91395)}

Ejemplares localizados con entrada en catálogo en línea (4):

- Londres, Victoria and Albert Museum, Dyce 26.D.30 (ejemplar digitalizado en EEBO)

- Middletown, Wesleyan University, E3

- New Haven, Yale University, He43 22Hn

4. The rogue: or, The life of Guzman de Alfarache. Written in Spanish by Matheo Aleman, servant to his Catholike Majestie, and borne in Sevill. To which is added, the tragi-comedy of Calisto and Melibea, represented in Celestina, London, Richard Badger, 1633-1634. Vendido por Robert Allot (Mab1634) ${ }^{49}$ : James Mabbe (trad.)

Principales repertorios on-line: USTC (3017309), ESTC (S1762) ${ }^{50}$

Ejemplares localizados con entrada en catálogo en línea (31):

- Austin, University of Texas at Austin, PQ 6272 E5 M3 1633 (ejemplar mútilo de las páginas [2]-[3] del final)

- Berkeley, University of California-Berkeley, PQ6272 .E5 1634

- Bryn Mawr, Bryn Mawr College, 863 Al2gE q (ejemplar mútilo de la parte correspondiente a Celestina)

- Cambridge (EE. UU.), Harvard University, Keats EC8 K2262 R622ac (ejemplar mútilo, las páginas 217-218 y 227-228 están incompletas)

49.- La parte correspondiente a Celestina tiene su propia portada con pie de imprenta "LONDON I Printed by J. B. And are to be sold by I Robert Allot at the Signe of the Beare I in Pauls church-yard. 1631.», idéntico al de Mab1631a, ya que se trata de los ejemplares que no se habían vendido de esa edición, reaprovechados (véase la introducción a Mabbe: 1972: 25).

50.- El ejemplar del Wheaton College que consignan tanto el USTC como el ESTC parece ser un libro digital, no físico, y además de la edición Mab1631a, porque no encuentro rastro de un ejemplar de Mab1634 en el catálogo. Tampoco he podido confirmar la presencia de los dos ejemplares de, respectivamente, Christ Church College y Magdalen College en Oxford que consignan tanto el USTC como el ESTC. Finalmente, el ejemplar de la University of Houston consignado por el ESTC parece ser una vez más un ejemplar electrónico, no físico. 
- Cambridge (EE. UU.), Harvard University, Keats EC8 K2262 Zz634a (portada mutilada y restaurada)

- Cambridge, University of Cambridge, Hisp.4.63.1

- Cambridge, University of Cambridge, Syn.4.63.46 (ejemplar mútilo de la parte correspondiente a Celestina)

- Cambridge, Trinity College (University of Cambridge), VI.12.26 (ejemplar mútilo de todas las hojas en blanco)

- Chapel Hill, University of North Carolina, PQ6272 .E5 M3 1634 (ejemplar mútilo de la parte correspondiente a Celestina)

- Chicago, University of Chicago, PQ6272.E7G8M203

- Chicago, Newberry Library, Case Y 1565 .A362

- Columbus, Ohio State University, PQ6272.E5 M2 1633 (ejemplar digitalizado en Hathi Trust y Google Books, pero mútilo de la parte correspondiente a Celestina)

- Durham, University of Durham, SB+ 0839

- Edimburgo, Advocates Library, Abbotsford

- Edimburgo, National Library of Scotland, Newb.3875 (ejemplar mútilo de la parte correspondiente a Celestina)

- Filadelfia, Penn State University, PQ 6272 E5 M3 1634 (ejemplar mútilo de cuatro hojas del cuadernillo A y de las hojas Eee2 y Eee5)

- Londres, British Library, 635.1.12.

- Londres, University of London, Bf [Aleman] fol SR (ejemplar mútilo de la parte correspondiente a Celestina)

- New Haven, Yale University, He67 +37W

- Nueva York, New York Public Library, *KC 1634 (Aleman, M. Rogve $)^{51}$

- Oxford, English Faculty Library (University of Oxford), YH97.8ALE[Rog]

- Oxford, Bodleian Library, Antiq.d.E.1634.3 (ejemplar mútilo de la parte correspondiente a Celestina)

- San Marino (EE. UU.), Huntington Library, 93569 (ejemplar con algunas hojas restauradas, incluida la portada)

- San Marino (EE. UU.), Huntington Library, 612540 (ejemplar mútilo de la parte correspondiente a Celestina y con otras imperfecciones que han sido restauradas)

- San Marino (EE. UU.), Huntington Library, 93569

- San Marino (EE. UU.), Huntington Library, 114920:171 (fragment) 
(ejemplar mútilo; se conserva la portada de la segunda parte del Guzmán de Alfarache)

- Urbana, University of Illinois at Urbana-Champaign, Q. 863 AL2OV.EM1634

- Washington, Folger Shakespeare Library, STC 291 copy 1

- Washington, Folger Shakespeare Library, STC 291 copy 2 (ejemplar mútilo de la parte correspondiente a Celestina)

- Washington, Folger Shakespeare Library, STC 291 copy 3 (ejemplar mútilo de la parte correspondiente a Celestina)

- York, York Minster, I.F.21(1)

Ejemplares localizados sin entrada en catálogo en línea (3):

- Cornwall, Lanhydrock (National Trust)

- Innerpeffray, Innerpeffray Library, G4 (USTC)

- Wimborne, Kingston Lacy (National Trust)

5. The Spanish libertines: or, The lives of Justina, the country jilt; Celestina, the bawd of Madrid; and Estevanillo Gonzales, the most arch and comical of scoundrels. Londres, Samuel Bunchley, 1707 $(\text { Stev1707a) })^{52}$ : John Stevens (trad.) ${ }^{53}$

Principales repertorios on-line: USTC (N/A), ESTC (N23941)

Ejemplares localizados con entrada en catálogo en línea (16):

- Austin, University of Texas at Austin, Ak St47 707s

- Cambridge, University of Cambridge, Syn.7.70.30

- Chicago, University of Chicago, PR3717.S32S8 1707

- Chicago, Newberry Library, Case Y 7254.84

- Columbus, Ohio State University, PQ6267.E1 S7 1707

- $\quad$ Eugene, University of Oregon, PQ6267.E1 S7 1707

- Evanston, Northwestern University, 863.08 S844s

- Hamilton, McMaster University, B 17123

- Londres, British Library, 12491.f.30.

- Los Angeles, University of California, PQ6267 .E3S8 *

52.- Edición que, a falta de una comparación más detallada, existe en dos emisiones, una con pie de imprenta de Samuel Bunchley (Stev1707a) y otra con pie de imprenta de John Howe (Stev1707b).

53.- No se trata propiamente de una traducción, sino de una adaptación escénica bastante libre. 
- Milán, Università degli Studi di Milano, APICE A.F.AG.A. X400 051

- Oxford, University of Oxford, Vet. A4 e.2200

- París, Bibliothèque Nationale de France, Y2-69579

- Urbana, University of Illinois at Urbana-Champaign, X 860.8 ST4S170954

- Viena, Österreichische Nationalbibliothek, 23693-B (ejemplar digitalizado en ÖNB y Google Books)

- Washington, Folger Shakespeare Library, 259580

6. The Spanish libertines: or, The lives of Justina, the country jilt; Celestina, the bawd of Madrid; and Estevanillo Gonzales, the most arch and comical of scoundrels. Londres, John Howe, 1707 (Stev1707b): John Stevens (trad.)

Principales repertorios on-line: USTC (N/A), ESTC (N23941) Ejemplares localizados con entrada en catálogo en línea (3):

- Northampton, Smith College, 825 St44e 1707

- San Marino (EE. UU.), Huntington Library, 147852

- Tucson, University of Arizona, PQ6252 .S713

7. The life of Guzman d'Alfarache: or, The Spanish rogue. To which is added, the celebrated tragi-comedy, Celestina. In two volumes, Londres. Rebecca Bonwick, William Freeman, Timothy Goodwin, John Walthoe, Matthew Wotton, John Nicholson, Samuel Manship, Richard Parker, Benjamin Tooke y Ralph Smith, 1707-1708 (Sav1707) ${ }^{55}$ : John Savage (trad.) $)^{56}$

\section{Principales repertorios on-line: USTC (N/A), ESTC (T86354) 57}

\section{4.- Es el ejemplar que el ESTC atribuye a Stev1709a.}

55.- Edición en dos volúmenes. La adaptación de Celestina se encuentra en el segundo, por lo que solo consigno los ejemplares conservados de este último y sus digitalizaciones. Lo mismo que en el caso de Mab1634, la parte de Celestina tiene su propia portada con año de impresión 1707, en lugar del año 1708 que figura en la portada del primer volumen. Dados los antecedentes de Mab1634, y puesto que no me consta ningún estudio al respecto, esta circunstancia merecería ser estudiada para saber si hubo una edición exenta de esta versión de Celestina en 1707 y para explicar por qué el primer volumen tiene fecha de impresión posterior al segundo.

56.- No se trata propiamente de una traducción, sino de una adaptación escénica bastante libre.

57.- El ejemplar de la Boston Public Library consignado por el ESTC parece ser un libro electrónico, no físico, mientras que el de la University of Virginia parece ser un microfilm. 
Ejemplares localizados con entrada en catálogo en línea (34):

- Aberystwyth, Llyfrgell Genedlaethol Cymru (National Library of Wales), PQ 6272 E5

- Ames, lowa State University, PQ6272.E5 A8

- Austin, University of Texas, PQ 6272 E5 A8

- Baton Rouge, Louisiana State University, PQ6272 .E5 A8

- Berkeley, University of California, PQ6272 .E5 1708

- Cambridge (EE. UU.), Harvard University, Span 5107.30*

- Chicago, Newberry Library, Case Y 1565 .A365

- Columbia, University of Missouri, PQ6272.E5 A8 17082

- Columbus, Ohio State University, PQ6272.E5 S2 v.2

- Dublín, Trinity College, W.L.4 [Vol.2?]

- Durham (EE. UU.), Duke University, PQ6272.E5 A8 1708 v.2 .c.1 (ejemplar digitalizado en Archive.org y Hathi Trust)

- Edimburgo, Advocates Library, Abbotsford

- Edimburgo, National Library of Scotland, [Ag].5/1.15-16

- Filadelfia, University of Pennsylvania, PQ6272.E5 A94 1707

- Hamilton, McMaster University, B 14103

- Halberstadt, Gleimhaus Museum, C 6893

- Houston, Rice University, PQ6272.E5 S3

- Leeds, University of Leeds, Brotherton Collection Lt ALE

- Londres, British Library, 12490.e.5.

- Londres, British Library, G.17678-79.

- Los Angeles, University of California, PQ6272.A3 E5 1708

- Notre Dame, University of Notre Dame, PQ 6272 .E5 A8

- Nottingham, University of Nottingham, PQ6272.A3.D07

- Nueva York, Columbia University, B86 C3358

- Oxford, Bodleian Library, Vet. A4 e.849 (v. 2)

- Oxford, Bodleian Library, Dunston B 51 (v. 2) (ejemplar dañado; mútilo de la parte correspondiente a Celestina)

- Oxford, Taylor Institution (University of Oxford), VET.SPAN.II.B.9 (v.2) (ejemplar digitalizado en Digital Books y en Google Books)

- Pretoria, National Library of South Africa, G.1.a.16-17

Igualmente, el ejemplar asignado a New York University por el ESTC parece ser una errata por el ejemplar de Columbia University, ubicada también en Nueva York.

58.- Es probable que este ejemplar sea el que el ESTC consigna como en la New York University, ya que no he podido encontrar ningún ejemplar en su catálogo. 
- San Marino (EE. UU.), Huntington Library, 111894

- State College, Penn State University, PQ6427.E56 1707

- Urbana, University of Illinois at Urbana-Champaign, X 863 AL2OV. E1708

- Viena, Universität Wien, I-170121/1

- Washington, Folger Shakespeare Library, PQ6272.A3E6 1708 Cage

- Washington, Library of Congress, PQ6272.E5 A8

Ejemplares localizados sin entrada en catálogo en línea (2):

- Belton, Belton House (National Trust)

- Kedleston, Kedleston Hall (National Trust)

8. The Spanish libertines: or, The lives of Justina, the country jilt; Celestina, the bawd of Madrid; and Estevanillo Gonzales, the most arch and comical of scoundrels. Londres, James Woodward, 1709 (Stev1709a) ${ }^{59}$ : John Stevens (trad.)

Principales repertorios on-line: USTC (N/A), ESTC (N37000)

Ejemplares localizados con entrada en catálogo en línea (3):

- Chicago, University of Chicago, PR3717.S32S8 1709

- Chicago, Newberry Library, Case Y 1565 .S921

- Washington, Folger Shakespeare Library, PR3717.S29S7 Cage

9. The Spanish libertines: or, The lives of Justina, the country jilt; Celestina, the bawd of Madrid; and Estevanillo Gonzales, the most arch and comical of scoundrels. Londres, Ebenezer Tracey, 1709 (Stev1709b): John Stevens (trad.)

Principales repertorios on-line: USTC (N/A), ESTC (-)

Ejemplares localizados con entrada en catálogo en línea (2):

- Cambridge (EE.UU.), Harvard University, EC7.St475.707sab

- New Haven, Yale University, He51 St47 


\section{Obras citadas}

Baldelli, Ignazio (1950), "Girolamo Claricio editore della Celestina», Giornale storico della letteratura italiana, 127, pp. 111-116.

Barth, Kaspar von (2006), Pornoboscodidascalus Latinus (1624). Kaspar Barth's Neo-Latin Translation of "Celestina», ed. Enrique Fernández Rivera, Chapel Hill, University of North Carolina.

BeARDSLEY, Theodore (1981), "The Lowlands Printings of Celestina (15391601)», Celestinesca, 5.1, pp. 7-11. <https://doi.org/10.7203/Celestinesca.5.19526>.

- (2005), "Early Editions of Celestina at The Hispanic Society of America», en La Celestina 1499-1999: Selected Papers from the International Congress in Commemoration of the Quincentennial Anniversary of "La Celestina", New York, November 17-19, 1999, ed. Ottavio Di Camillo y John O’Neill, New York, Hispanic Seminary of Medieval Studies, pp. 7-17.

BeHIELs, Lieve, y Katherine V. Kish (eds.), (2005), "Celestina»: An Annotated Edition of the First Dutch Translation (Antwerp, 1550), Leuven, Leuven University Press.

BeRndT-Kelley, Erna (1988), "Elenco de ejemplares de ediciones tempranas del texto original y de traducciones de la obra de Fernando de Rojas en Canadá, Estados Unidos y Puerto Rico», Celestinesca, 12.1, pp. 9-34. <https://doi.org/10.7203/Celestinesca.12.19670>.

BP16, Bibliographie des éditions parisiennes du 16e siècle, base de datos bibliográfica. Disponible on-line <https://bp16.bnf.fr>.

BraulT, Gerard J. (1960), "English Translations of the Celestina in the Sixteenth Century», Hispanic Review, 28.4, pp. 301. <https://doi. org/10.2307/471194>.

- (ed.), (1963), "Célestine»: A Critical Edition of the First French Translation (1527) of the Spanish Classic «La Celestina», Detroit - Toronto, Wayne State University Press - Ambassador Books.

Carmona, Fernando (2007), "La recepción de La Celestina en Alemania en el siglo XVI», tesis doctoral, Freiburg, Universität Freiburg. Disponible on-line <http://nbn-resolving.de/urn:nbn:ch:rero-002-100612>.

Cornejo, Rafael (1977), "Bibliografía de La Celestina», en "La Celestina»y su contorno social: Actas del I Congreso Internacional sobre "La Celestina", ed. Manuel Criado de Val, Madrid, Hispam, pp. 552-584.

Drysdall, Denis L. (1970), "La Celestina en France de 1521 à 1812», tesis doctoral, Paris, Université de la Sorbonne.

EDIT16, Censimento nazionale delle edizioni italiane del XVI secolo, base de datos bibliográfica. Disponible on-line <http://edit16.iccu.sbn.it>.

ESTC, English Short Title Catalogue, base de datos bibliográfica. Disponible on-line <http://estc.bl.uk>. 
Fernández González, Emilio (2005), «La Celestina en alemán: un ejemplar único en España de la Biblioteca Histórica de la Universidad Complutense», Pecia Complutense, 2.2, pp. 1-8.

Genske, Sylvia Simpson (1978), "La Celestina in Translation before 1530», tesis doctoral, New York, New York University.

GIRALDO, Federica (2013), "Transcripción y estudio paleografico de la primera traducción italiana de La Celestina [Bib. Marciana -DRAMM. 2988.5-]», trabajo de final de grado, Venecia, Università Ca' Foscari. Disponible on-line $<$ http://dspace.unive.it/handle/10579/3615>.

Givanel Mas, Juan (1919), "Contribución al estudio bibliográfico de la Celestina y descripción de un rarísimo ejemplar de dicha obra", Revista Crítica Hispano-Americana, 5.3-4, pp. 77-121.

Gómez Blanco, Emilio F. B. (2001), "Algunas notas sobre la recepción de Celestina en los siglos XVI y XVII", en "Celestina»: Recepción y herencia de un mito literario, ed. Gregorio Torres Nebrera, Cáceres, Universidad de Extremadura, pp. 17-50.

Hordognez, Alphonso (1973), An Edition of the First Italian Translation of the "Celestina", ed. Kathleen V. Kish, Chapel Hill, University of North Carolina Press.

INFANTES, Víctor (2007), «El laberinto cronológico y editorial de las primitivas impresiones de Celestina (1497-1514). Con una Marginalia bibliographica al cabo", en Actas del Simposio Internacional 1502-2002: Five Hundred Years of Fernando de Rojas' "Tragicomedia de Calisto y Melibea", ed. Juan Carlos Conde, Nueva York, Hispanic Seminary of Medieval Studies, pp. 3-87.

KisH, Kathleen V. (1971), "An Edition of the First Italian Translation of the Celestina", tesis doctoral, Madison, University of Wisconsin-Madison.

- (1989), "Celestina en Amberes en el siglo XVI», en Actas del IX Congreso de la Asociación Internacional de Hispanistas, ed. Sebastian Neumeister, Madrid - Frankfurt, Iberoamericana - Vervuert, pp. 525-534.

Kish, Kathleen V., y Ursula Ritzenhoff (1980), «The Celestina Phenomenon in sixteenth-century Germany: Christof Wirsung's Translations of 1520 and 1534», Celestinesca, 4.2, pp. 9-18. <https://doi.org/10.7203/ Celestinesca.4.19512>.

KrapF, Eugenio (1900), «Una reseña de las principales traducciones de la Celestina», en La Celestina: Tragicomedia de Calisto y Melibea, ed. Marcelino Menéndez y Pelayo, Vigo, E. Krapf, pp. LX-XCIX.

KVK, Karlsruher Virtueller Katalog, metacatálogo bibliográfico. Disponible on-line $<$ https://kvk.bibliothek.kit.edus.

LaCARRA, María Jesús, y Ana Milagros Jiménez Ruiz (2021), «Testimonios recuperados de La Celestina: una nueva edición de la Tragicomedia de Calisto y Melibea hallada en la Biblioteca estatal de Ulm», Celestinesca, 45, pp. 97-124. <https://doi.org/10.7203/Celestinesca.45.21885>. 
LAmpugnani, Raffaele (1983), "Il metodo e lo stile di Alphonso Hordognez nella prima traduzione della Celestina», trabajo de final de máster, Adelaida, Flinders University of South Australia.

- (1992), "Traveller's Tails: Subjective Textual Changes in the First Italian Translation of La Celestina», Romance Studies, 11, pp. 85-96.

- (2015), La prima traduzione italiana de "La Celestina": primo commento linguistico e critico agli inizi del Cinquecento, Firenze, Leo S. Olschki.

LAVARDIN, Jacques de (1974), "La Célestine». In the French Translation of 1578 by Jacques de Lavardin, ed. Denis L. Drysdall, London, Tamesis Books Limited.

Lysy, Katia (1985), "La traduzione romana del 1506 della Celestina», tesis doctoral, Roma, Università degli Studi di Roma, La Sapienza.

MabBe, James (1972), Celestine or the Tragick-Comedie of Calisto and Melibea, ed. Guadalupe Martínez Lacalle, London, Tamesis Books Limited.

- (1987), Celestina (bilingual edition), ed. Dorothy Sherman Severin, Warminster, Aris \& Phillips.

- (2013), James Mabbe, The Spanish Bawd, ed. José María Pérez Fernández, London, Modern Humanities Research Association. Disponible on-line <https://www.jstor.org/stable/j.ctt4cg909>.

Melzi, Robert C. (2000), "Celestina Italian Style», Rivista di Studi Italiani, 18.2, pp. 32-43.

Menéndez y Pelayo, Marcelino (1943), "Las traducciones de La Celestina», en Orígenes de la novela. III- Cuentos y novelas Cortas. "La Celestina", Madrid, Consejo Superior de Investigaciones Científicas, pp. 411-433.

Miguel y Canuto, Juan-Carlos de (2003), "Sosta nel labirinto: bilancio bibliografico sulla prima traduzione italiana di La Celestina», Studi e Problemi di Critica Testuale, 67, pp. 71-108.

MiragliotTa, Elisa (2017), «Transcripción paleográfica y análisis de la segunda traducción italiana de la tragicomedia La Celestina [Biblioteca Nazionale Marciana DRAMM 0123]», trabajo de final de grado, Venezia, Università Ca' Foscari. Disponible on-line <http://dspace.unive.it/handle/10579/9703>.

Morreale, Margherita (1990), "Apuntes bibliográficos para el estudio de la presencia de La Celestina en Italia», Revista de literatura, 52.104, pp. 539-543.

NewTon, Jeremy (1974), «Two Eighteenth-Century English Adaptations of the Celestina: Celestina: Or the Spanish Bawd: A Tragi-Comedy; and The Bawd of Madrid", tesis doctoral, London, Quenn Mary, University of London. Disponible on-line <http://qmro.qmul.ac.uk/xmlui/ handle/123456789/1613>.

OlivetTo, Georgina (1998), «Ejemplares de Celestina de la colección Foulché-Delbosc en la Biblioteca Nacional de la República Argentina", Celestinesca, 22.1, pp. 67-74. <https://doi.org/10.7203/Celestinesca.22.19900>. 
Paolini, Devid (2010), "Ediciones de La Celestina anteriores al siglo XIX en la Biblioteca Nacional de España», Revista de Literatura Medieval, 22, pp. 351-359. Disponible on-line <https://ebuah.uah.es/dspace/handle/10017/10466>

Penney, Clara (1954), The Book called "Celestina" in the Library of the Hispanic Society of America, New York, Hispanic Society of America.

Pettegree, Andrew et al. (eds.), (2007), French Vernacular Books: Books Published in the French Language before 1601 / Livres vernaculaires français: livres imprimés en français avant 1601, 2 vols., Leiden - Boston, Brill.

Piñero, Leonor (1984), "La difusión de la Celestina en Italia», Gades, 12, pp. 315-336.

Rastell, John (2001), Interlude of Calisto and Melebea, ed. Rubén Tostado González, Salamanca, Universidad de Salamanca.

Saguar García, Amaranta (2012), "Un catálogo actualizado de las ediciones quinientistas de la traducción italiana de Celestina», trabajo inconcluso inédito, CORE: Open Access for the Humanities. Disponible on-line <https://hcommons.org/deposits/item/hc:22129>.

- (2015), "¿Hubo otra traducción quinientista de Celestina al italiano?», Celestinesca, 39, pp. 53-60. <https://doi.org/10.7203/Celestinesca.39.20177>.

- (2016), «¿Cuestión de moda? La desaparición de la traducción italiana de Celestina del mercado editorial en la segunda mitad del siglo XVI», en Grandes y pequeños de la literatura medieval y renacentista, ed. Emilio Blanco, Salamanca, SEMYR, pp. 625-642.

Saguar García, Amaranta (2018), "El texto base de las traducciones quinientistas de Celestina al alemán (1520 y 1534)», Bulletin of Hispanic Studies, 95.2, pp. 127-144. <https://doi.org/10.3828/bhs.2018.8>.

- (2020), "Hacia un censo completo unificado de los ejemplares conservados de Celestina (I): ejemplares de ediciones en castellano localizables en línea», Celestinesca, 44, pp. 265-318. <https://doi.org/10.7203/ Celestinesca.44.19435>.

SCOLES, Emma (1961), "Note sulla prima traduzione italiana della Celestina», Studj Romanzi, 33, pp. 153-217.

SCOLES, Emma (1964), «La prima traduzione italiana della Celestina: repertorio bibliografico", Studi di letteratura spagnola, I, pp. 209-230.

Serrano, Florence (2008), "La Celestina en la Francia del Renacimiento y del Siglo de Oro: Texto y contexto, difusión y fortuna", Celestinesca, 32.1, pp. 265-278. <https://doi.org/10.7203/Celestinesca.32.20118>.

Simone, Carla (1991), "La prima traduzione italiana della Celestina ed i suoi rapporti con le edizioni primitive in lingua spagnola», tesis doctoral, Roma, Università degli Studi di Roma, La Sapienza.

Snow, Joseph T. (2001), «Historia de la recepción de Celestina: 1499-1822. II (1499-1600)», Celestinesca, 25, pp. 199-282.<https://doi.org/10.7203/ Celestinesca.25.19983>. 
STCN, Short Title Catalogue Netherlands, base de datos bibliográfica. Disponible on-line $<$ https://picarta.oclc.org $>$.

Ubaldi, Danila (1993), «Le versioni italiane della Celestina. Problematiche di traduzione», tesis doctoral, Roma, Università degli Studi di Roma, La Sapienza.

USTC, Universal Short Title Catalogue, base de datos bibliográfica. Disponible on-line $<$ https://www.ustc.ac.uk $>$.

VD16, Verzeichnis der im deutschen Sprachraum erschienenen Drucke des 16. Jahrhunderts, base de datos bibliográfica. Disponible on-line <http:// www.gateway-bayern.de/index_vd16.html>.

VD17, Verzeichnis der im deutschen Sprachraum erschienenen Drucke des 17. Jahrhunderts, base de datos bibliográfica. Disponible on-line <http:// www.vd17.de>.

WaGner, Christine (1987), "La première traduction italienne de La Celestina par Alphonso Hordognez, Rome, 1506», tesis doctoral, Lille, Université de Lille III.

Wirsung, Christof (1984), Die Celestina-Übersetzungen von Christof Wirsung: "Ain hipsche Tragedia» (Augsburg 1520), "Ainn recht liepliches Buechlin» (Augsburg 1534), ed. Kathleen V. Kish y Ursula Ritzenhoff, Hildesheim, G. Olms Verlag. 